\title{
Measurement of the partial decay width $R_{b}^{0}=\Gamma_{b \bar{b}} / \Gamma_{h a d}$ of the $Z$ with the DELPHI detector at LEP
}

\author{
DELPHI Collaboration
}

\begin{abstract}
The partial decay width of the $Z$ to $b \bar{b}$ quark pairs has been measured by the DELPHI detector at LEP. B-hadrons, containing $b$-quarks, were tagged by several methods using tracks with large impact parameters to the primary vertex complemented sometimes by event shape variables or using leptons with high transverse momentum relative to the hadron.

In order to reduce the systematic uncertainties, in all methods the $b$-tagging efficiency has been extracted directly from the data. Combining all methods, the value:

$$
\frac{\Gamma_{b \bar{b}}}{\Gamma_{\text {had }}}=0.2216 \pm 0.0016(\text { stat.) } \pm 0.0021 \text { (syst.) }
$$

was found, where the $c \bar{c}$ production fraction was fixed to its Standard Model value.
\end{abstract}


P.Abreu ${ }^{21}$, W.Adam ${ }^{50}$, T.Adye ${ }^{37}$, E.Agasi $^{31}$, I.Ajinenko ${ }^{42}$, R.Aleksan ${ }^{39}$, G.D.Alekseev ${ }^{16}$, R.Alemany ${ }^{49}$, P.P.Allport ${ }^{22}$, S.Almehed ${ }^{24}$, U.Amaldi ${ }^{9}$, S.Amato ${ }^{47}$, A.Andreazza ${ }^{28}$, M.L.Andrieux ${ }^{14}$, P.Antilogus ${ }^{9}$, W-D.Apel ${ }^{17}$, Y.Arnoud ${ }^{39}$, B.Asman ${ }^{44}$, J-E.Augustin ${ }^{19}$, A.Augustinus ${ }^{9}$, P.Baillon ${ }^{9}$, P.Bambade ${ }^{19}$, F.Barao $^{21}$, R.Barate $^{14}$, M.Barbi ${ }^{47}$, D.Y.Bardin ${ }^{16}$, A.Baroncelli ${ }^{40}$, O.Barring ${ }^{24}$, J.A.Barrio ${ }^{26}$, W.Bartl ${ }^{50}$, M.J.Bates ${ }^{37}$, M.Battaglia ${ }^{15}$, M.Baubillier ${ }^{23}$, J.Baudot ${ }^{39}$, K-H.Becks ${ }^{52}$, M.Begalli ${ }^{6}$, P.Beilliere ${ }^{8}$, Yu.Belokopytov ${ }^{9,53}$, A.C.Benvenuti $^{5}$, M.Berggren ${ }^{47}$, D.Bertrand ${ }^{2}$, F.Bianchi ${ }^{45}$, M.Bigi ${ }^{45}$, M.S.Bilenky ${ }^{16}$, P.Billoir ${ }^{23}$, D.Bloch ${ }^{10}$, M.Blume $^{52}$, S.Blyth ${ }^{35}$, T.Bolognese ${ }^{39}$, M.Bonesini ${ }^{28}$, W.Bonivento ${ }^{28}$, P.S.L.Booth ${ }^{22}$, G.Borisov ${ }^{42}$, C.Bosio $^{40}$, S.Bosworth $^{35}$, O.Botner ${ }^{48}$, E.Boudinov ${ }^{31}$, B.Bouquet ${ }^{19}$, C.Bourdarios ${ }^{9}, \quad$ T.J.V.Bowcock ${ }^{22}$, M.Bozzo ${ }^{13}$, P.Branchini $^{40}$, K.D.Brand ${ }^{36}$, T.Brenke ${ }^{52}$, R.A.Brenner ${ }^{15}$, C.Bricman ${ }^{2}$, L.Brillault ${ }^{23}$, R.C.A.Brown ${ }^{9}$, P.Bruckman $^{18}$, J-M.Brunet ${ }^{8}$, L.Bugge ${ }^{33}$, T.Buran ${ }^{33}$, T.Burgsmueller ${ }^{52}$, P.Buschmann ${ }^{52}$, A.Buys ${ }^{9}$, S.Cabrera ${ }^{49}$, M.Caccia ${ }^{28}$, M.Calvi $^{28}$, A.J.Camacho Rozas ${ }^{41}$, T.Camporesi ${ }^{9}$, V.Canale ${ }^{38}$, M.Canepa ${ }^{13}$, K.Cankocak $^{44}$, F.Cao $^{2}$, F.Carena $^{9}$, L.Carroll ${ }^{22}$, C.Caso ${ }^{13}$, M.V.Castillo Gimenez ${ }^{49}$, A.Cattai ${ }^{9}$, F.R.Cavallo ${ }^{5}$, L.Cerrito $^{38}$, V.Chabaud $^{9}$, Ph.Charpentier $^{9}$, L.Chaussard ${ }^{25}$, J.Chauveau ${ }^{23}$, P.Checchia ${ }^{36}$, G.A.Chelkov ${ }^{16}$, M.Chen ${ }^{2}$, R.Chierici ${ }^{45}$, P.Chliapnikov $^{42}$, P.Chochula ${ }^{7}$, V.Chorowicz ${ }^{9}$, J.Chudoba ${ }^{30}$, V.Cindro ${ }^{43}$, P.Collins ${ }^{9}$, J.L.Contreras ${ }^{19}$, R.Contri ${ }^{13}$, E.Cortina ${ }^{49}$, G.Cosme ${ }^{19}$, F.Cossutti ${ }^{46}$, H.B.Crawley ${ }^{1}$, D.Crennell ${ }^{37}$, G.Crosetti ${ }^{13}$, J.Cuevas Maestro $^{34}$, S.Czellar ${ }^{15}$, E.Dahl-Jensen ${ }^{29}$, J.Dahm ${ }^{52}$, B.Dalmagne ${ }^{19}$, M.Dam ${ }^{29}$, G.Damgaard ${ }^{29}$, P.D.Dauncey ${ }^{37}$, M.Davenport ${ }^{9}$, W.Da Silva ${ }^{23}$, C.Defoix ${ }^{8}$, A.Deghorain ${ }^{2}$, G.Della Ricca ${ }^{46}$, P.Delpierre ${ }^{27}$, N.Demaria $^{35}$, A.De Angelis ${ }^{9}$, W.De Boer ${ }^{17}$, S.De Brabandere ${ }^{2}$, C.De Clercq ${ }^{2}$ C.De La Vaissiere ${ }^{23}$, B.De Lotto ${ }^{46}$, A.De Min ${ }^{36}$, L.De Paula ${ }^{47}$, C.De Saint-Jean ${ }^{39}$, H.Dijkstra ${ }^{9}$, L.Di Ciaccio ${ }^{38}$, F.Djama $^{10}$, J.Dolbeau $^{8}$, M.Donszelmann ${ }^{9}$, K.Doroba ${ }^{51}$, M.Dracos ${ }^{10}$, J.Drees ${ }^{52}$, K.-A.Drees ${ }^{52}$, M.Dris ${ }^{32}$, Y.Dufour ${ }^{9}$, D.Edsall $^{1}$, R.Ehret ${ }^{17}$, G.Eigen ${ }^{4}$, T.Ekelof ${ }^{48}$, G.Ekspong ${ }^{44}$, M.Elsing ${ }^{52}$, J-P.Engel ${ }^{10}$, N.Ershaidat ${ }^{23}$, B.Erzen $^{43}$, M.Espirito Santo ${ }^{21}$, E.Falk ${ }^{24}$, D.Fassouliotis ${ }^{32}$, M.Feindt ${ }^{9}$, A.Fenyuk ${ }^{42}$, A.Ferrer ${ }^{49}$, T.A.Filippas ${ }^{32}$, A.Firestone $^{1}$, P.-A.Fischer ${ }^{10}$, H.Foeth ${ }^{9}$, E.Fokitis ${ }^{32}$, F.Fontanelli ${ }^{13}$, F.Formenti ${ }^{9}$, B.Franek ${ }^{37}$, P.Frenkiel ${ }^{8}$, D.C.Fries ${ }^{17}$, A.G.Frodesen ${ }^{4}$, R.Fruhwirth ${ }^{50}$, F.Fulda-Quenzer ${ }^{19}$, J.Fuster ${ }^{49}$, A.Galloni ${ }^{22}$, D.Gamba ${ }^{45}$, M.Gandelman ${ }^{6}$, C.Garcia ${ }^{49}$, J.Garcia ${ }^{41}$, C.Gaspar $^{9}$, U.Gasparini ${ }^{36}$, Ph.Gavillet ${ }^{9}$, E.N.Gazis ${ }^{32}$, D.Gele ${ }^{10}$, J-P.Gerber ${ }^{10}$, M.Gibbs $^{22}$, R.Gokieli ${ }^{51}$, B.Golob ${ }^{43}$, G.Gopal $^{37}$, L.Gorn ${ }^{1}$, M.Gorski ${ }^{51}$, Yu.Gouz ${ }^{45,53}$, V.Gracco ${ }^{13}$, E.Graziani ${ }^{40}$, G.Grosdidier ${ }^{19}$, K.Grzelak ${ }^{51}$, S.Gumenyuk ${ }^{28,53}$, P.Gunnarsson ${ }^{44}$, M.Gunther ${ }^{48}$, J.Guy ${ }^{37}$, F.Hahn ${ }^{9}$, S.Hahn ${ }^{52}$, Z.Hajduk ${ }^{18}$, A.Hallgren ${ }^{48}$, K.Hamacher ${ }^{52}$, W.Hao ${ }^{31}$, F.J.Harris ${ }^{35}$, V.Hedberg ${ }^{24}$, R.Henriques $^{21}$, J.J.Hernandez ${ }^{49}$, P.Herquet ${ }^{2}$, H.Herr ${ }^{9}$, T.L.Hessing ${ }^{35}$, E.Higon ${ }^{49}$, H.J.Hilke ${ }^{9}$, T.S.Hill ${ }^{1}$, S-O.Holmgren $^{44}$, P.J.Holt ${ }^{35}$, D.Holthuizen ${ }^{31}$, S.Hoorelbeke ${ }^{2}$, M.Houlden ${ }^{22}$, J.Hrubec ${ }^{50}$, K.Huet ${ }^{2}$, K.Hultqvist ${ }^{44}$, J.N.Jackson ${ }^{22}$, R.Jacobsson ${ }^{44}$, P.Jalocha ${ }^{18}$, R.Janik ${ }^{7}$, Ch.Jarlskog ${ }^{24}$, G.Jarlskog ${ }^{24}$. P.Jarry ${ }^{39}$, B.Jean-Marie ${ }^{19}$, E.K.Johansson ${ }^{44}$, L.Jonsson ${ }^{24}$, P.Jonsson ${ }^{24}$, C.Joram ${ }^{9}$, P.Juillot ${ }^{10}$, M.Kaiser ${ }^{17}$, F.Kapusta ${ }^{23}$, K.Karafasoulis ${ }^{11}$, M.Karlsson ${ }^{44}$, E.Karvelas ${ }^{11}$, A.Katargin ${ }^{42}$, S.Katsanevas ${ }^{3}$, E.C.Katsoufis ${ }^{32}$, R.Keranen ${ }^{4}$, Yu.Khokhlov ${ }^{42}$, B.A.Khomenko ${ }^{16}$, N.N.Khovanski ${ }^{16}$, B.King ${ }^{22}$, N.J.Kjaer ${ }^{29}$, H.Klein ${ }^{9}$, A.Klovning ${ }^{4}$, P.Kluit ${ }^{31}$, B.Koene ${ }^{31}$, P.Kokkinias $^{11}$, M.Koratzinos ${ }^{9}$, K.Korcyl ${ }^{18}$, C.Kourkoumelis ${ }^{3}$, O.Kouznetsov ${ }^{13,16}$, P.-H.Kramer ${ }^{52}$, M.Krammer $^{50}$, C.Kreuter ${ }^{17}$, I.Kronkvist ${ }^{24}$, Z.Krumstein ${ }^{16}$, W.Krupinski ${ }^{18}$, P.Kubinec ${ }^{7}$, W.Kucewicz ${ }^{18}$, K.Kurvinen $^{15}$, C.Lacasta $^{49}$, I.Laktineh ${ }^{25}$, S.Lamblot ${ }^{23}$, J.W.Lamsa ${ }^{1}$, L.Lanceri ${ }^{46}$, D.W.Lane ${ }^{1}$, P.Langefeld ${ }^{52}$, I.Last $^{22}$, J-P.Laugier ${ }^{39}$, R.Lauhakangas ${ }^{15}$, F.Ledroit ${ }^{14}{ }^{\text {, }}$ V.Lefebure $^{2}$, C.K.Legan ${ }^{1}$, R.Leitner ${ }^{30}$, Y.Lemoigne ${ }^{39}$, J.Lemonne $^{2}$, G.Lenzen ${ }^{52}$, V.Lepeltier ${ }^{19}$, T.Lesiak ${ }^{36}$, D.Liko ${ }^{50}$, R.Lindner ${ }^{52}$, A.Lipniacka ${ }^{36}$, I.Lippi ${ }^{36}$, B.Loerstad ${ }^{24}$, J.G.Loken ${ }^{35}$, J.M.Lopez ${ }^{41}$, D.Loukas ${ }^{11}$, P.Lutz ${ }^{39}$, L.Lyons ${ }^{35}$, J.MacNaughton ${ }^{50}$, G.Maehlum ${ }^{17}$, A.Maio $^{21}$, V.Malychev ${ }^{16}$, F.Mandl ${ }^{50}$, J.Marco ${ }^{41}$, R.Marco ${ }^{41}$, B.Marechal ${ }^{47}$, M.Margoni ${ }^{36}$, J-C.Marin ${ }^{9}$, C.Mariotti $^{40}$, A.Markou ${ }^{11}$, T.Maron ${ }^{52}$, C.Martinez-Rivero ${ }^{41}$, F.Martinez-Vidal ${ }^{49}$, S.Marti i Garcia ${ }^{49}$, J.Masik ${ }^{30}$, F.Matorras $^{41}$, C.Matteuzzi ${ }^{9}$, G.Matthiae ${ }^{38}$, M.Mazzucato ${ }^{36}$, M.Mc Cubbin ${ }^{9}$, R.Mc Kay ${ }^{1}$, R.Mc Nulty ${ }^{22}$, J.Medbo $^{48}$, M.Merk ${ }^{31}$, C.Meroni ${ }^{28}$, S.Meyer ${ }^{17}$, W.T.Meyer ${ }^{1}$, A.Miagkov ${ }^{42}$, M.Michelotto ${ }^{36}$, E.Migliore ${ }^{45}$, L.Mirabito $^{25}$, W.A.Mitaroff ${ }^{50}$, U.Mjoernmark ${ }^{24}$, T.Moa ${ }^{44}$, R.Moeller ${ }^{29}$, K.Moenig ${ }^{9}$, M.R.Monge ${ }^{13}$, P.Morettini $^{13}$, H.Mueller ${ }^{17}$, L.M.Mundim ${ }^{6}$, W.J.Murray ${ }^{37}$, B.Muryn ${ }^{18}$, G.Myatt ${ }^{35}$, F.Naraghi ${ }^{14}$, F.L.Navarria ${ }^{5}$, S.Navas $^{49}$, K.Nawrocki ${ }^{51}$, P.Negri ${ }^{28}$, W.Neumann ${ }^{52}$, N.Neumeister ${ }^{50}$, R.Nicolaidou ${ }^{3}$, B.S.Nielsen ${ }^{29}$, M.Nieuwenhuizen $^{31}$, V.Nikolaenko ${ }^{10}$, P.Niss ${ }^{44}$, A.Nomerotski ${ }^{36}$, A.Normand ${ }^{35}$, W.Oberschulte-Beckmann ${ }^{17}$, V.Obraztsov $^{42}$, A.G.Olshevski ${ }^{16}$, A.Onofre ${ }^{21}$, R.Orava ${ }^{15}$, K.Osterberg ${ }^{15}$, A.Ouraou ${ }^{39}$, P.Paganini $^{19}$, M.Paganoni $^{9}$, P.Pages $^{10}$, H.Palka ${ }^{18}$, Th.D.Papadopoulou ${ }^{32}$, K.Papageorgiou ${ }^{11}$, L.Pape ${ }^{9}$, C.Parkes ${ }^{35}$, F.Parodi ${ }^{13}$, A.Passeri ${ }^{40}$, M.Pegoraro ${ }^{36}$, H.Pernegger ${ }^{50}$, M.Pernicka ${ }^{50}$, A.Perrotta ${ }^{5}$, C.Petridou $^{46}$, A.Petrolini ${ }^{13}$, M.Petrovyck $^{28,53}$, H.T.Phillips ${ }^{37}$, G.Piana ${ }^{13}$, F.Pierre ${ }^{39}$, M.Pimenta ${ }^{21}$, M.Pindo ${ }^{28}$, S.Plaszczynski ${ }^{19}$, O.Podobrin ${ }^{17}$, M.E.Pol ${ }^{6}$, G.Polok ${ }^{18}$, P.Poropat ${ }^{46}$, V.Pozdniakov ${ }^{16}$, M.Prest ${ }^{46}$, P.Privitera ${ }^{38}$, N.Pukhaeva ${ }^{16}$, A.Pullia ${ }^{28}$, D.Radojicic ${ }^{35}$, S.Ragazzi ${ }^{28}$, H.Rahmani ${ }^{32}$, J.Rames ${ }^{12}$, P.N.Ratoff ${ }^{20}$, A.L.Read ${ }^{33}$, M.Reale ${ }^{52}$, P.Rebecchi $^{19}$, N.G.Redaelli ${ }^{28}$, M.Regler ${ }^{50}$, D.Reid ${ }^{9}$, P.B.Renton ${ }^{35}$, L.K.Resvanis ${ }^{3}$, F.Richard ${ }^{19}$, J.Richardson $^{22}$, J.Ridky $^{12}$, G.Rinaudo ${ }^{45}$, I.Ripp ${ }^{39}$, A.Romero ${ }^{45}$, I.Roncagliolo ${ }^{13}$, P.Ronchese ${ }^{36}$, L.Roos ${ }^{14}$, E.I.Rosenberg ${ }^{1}$, E.Rosso $^{9}$, P.Roudeau ${ }^{19}$, T.Rovelli ${ }^{5}$, W.Ruckstuhl ${ }^{31}$, V.Ruhlmann-Kleider ${ }^{39}$, A.Ruiz ${ }^{41}$, K.Rybicki ${ }^{18}$, H.Saarikko ${ }^{15}$, Y.Sacquin ${ }^{39}$, A.Sadovsky ${ }^{16}$, G.Sajot $^{14}$, J.Salt ${ }^{49}$, J.Sanchez ${ }^{26}$, M.Sannino ${ }^{13}$, M.Schimmelpfennig ${ }^{17}$, H.Schneider ${ }^{17}$, U.Schwickerath ${ }^{17}$, M.A.E.Schyns ${ }^{52}$, G.Sciolla ${ }^{45}$, F.Scuri ${ }^{46}$, P.Seager ${ }^{20}$, Y.Sedykh ${ }^{16}$, A.M.Segar ${ }^{35}$,

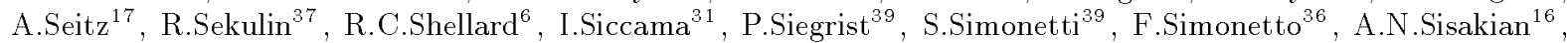
B.Sitar ${ }^{7}$, T.B.Skaali ${ }^{33}$, G.Smadja ${ }^{25}$, N.Smirnov ${ }^{42}$, O.Smirnova ${ }^{16}$, G.R.Smith ${ }^{37}$, O.Solovianov ${ }^{42}$, R.Sosnowski ${ }^{51}$, D.Souza-Santos ${ }^{6}$, T.Spassov ${ }^{21}$, E.Spiriti ${ }^{40}$, P.Sponholz ${ }^{52}$, S.Squarcia ${ }^{13}$, C.Stanescu $^{40}$, S.Stapnes ${ }^{33}$, I.Stavitski ${ }^{36}$, F.Stichelbaut $^{9}$, A.Stocchi ${ }^{19}$, J.Strauss ${ }^{50}$, R.Strub ${ }^{10}$, B.Stugu ${ }^{4}$, M.Szczekowski ${ }^{51}$, M.Szeptycka ${ }^{51}$, T.Tabarelli ${ }^{28}$, 
J.P.Tavernet ${ }^{23}$, O.Tchikilev ${ }^{42}$, A.Tilquin ${ }^{27}$, J.Timmermans ${ }^{31}$, L.G.Tkatchev ${ }^{16}$, T.Todorov ${ }^{10}$, D.Z.Toet $^{31}$, A.Tomaradze $^{2}$, B.Tome ${ }^{21}$, A.Tonazzo ${ }^{28}$, L.Tortora ${ }^{40}$, G.Transtromer ${ }^{24}$, D.Treille ${ }^{9}$, W.Trischuk ${ }^{9}$, G.Tristram ${ }^{8}$, A.Trombini ${ }^{19}$, C.Troncon ${ }^{28}$, A.Tsirou ${ }^{9}, \quad$ M-L.Turluer ${ }^{39}$, I.A.Tyapkin ${ }^{16}$, M.Tyndel ${ }^{37}, \quad$ S.Tzamarias ${ }^{22}$, B.Ueberschaer ${ }^{52}$, O.Ullaland ${ }^{9}$, V.Uvarov ${ }^{42}$, G.Valenti ${ }^{5}$, E.Vallazza ${ }^{9}$, C.Vander Velde ${ }^{2}$, G.W.Van Apeldoorn ${ }^{31}$, P.Van Dam ${ }^{31}$, W.K.Van Doninck ${ }^{2}$, J.Van Eldik ${ }^{31}$, N.Vassilopoulos ${ }^{35}$, G.Vegni ${ }^{28}$, L.Ventura ${ }^{36}$, W.Venus ${ }^{37}$, F.Verbeure $^{2}$, M.Verlato ${ }^{36}$, L.S.Vertogradov ${ }^{16}$, D.Vilanova ${ }^{39}$, P.Vincent ${ }^{25}$, L.Vitale ${ }^{46}$, E.Vlasov $^{42}$, A.S.Vodopyanov ${ }^{16}$, V.Vrba ${ }^{12}$, H.Wahlen ${ }^{52}$, C.Walck ${ }^{44}$, F.Waldner ${ }^{46}$, M.Weierstall ${ }^{52}$, P.Weilhammer ${ }^{9}$, C.Weiser $^{17}$, A.M.Wetherell ${ }^{9}$, D.Wicke ${ }^{52}$, J.H.Wickens ${ }^{2}$, M.Wielers ${ }^{17}$, G.R.Wilkinson ${ }^{35}$, W.S.C.Williams ${ }^{35}$, M.Winter $^{10}$, M.Witek ${ }^{18}$, K.Woschnagg ${ }^{48}$, K.Yip ${ }^{35}$, O.Yushchenko ${ }^{42}$, F.Zach ${ }^{25}$, A.Zaitsev ${ }^{42}$, A.Zalewska ${ }^{18}$, P.Zalewski ${ }^{51}$, D.Zavrtanik ${ }^{43}$, E.Zevgolatakos ${ }^{11}$, N.I.Zimin ${ }^{16}$, M.Zito ${ }^{39}$, D.Zontar ${ }^{43}$, R.Zuberi ${ }^{35}$, G.C.Zucchelli ${ }^{44}$, G.Zumerle ${ }^{36}$

\footnotetext{
${ }^{1}$ Ames Laboratory and Department of Physics, Iowa State University, Ames IA 50011, USA

${ }^{2}$ Physics Department, Univ. Instelling Antwerpen, Universiteitsplein 1, B-2610 Wilrijk, Belgium and IIHE, ULB-VUB, Pleinlaan 2, B-1050 Brussels, Belgium

and Faculté des Sciences, Univ. de l'Etat Mons, Av. Maistriau 19, B-7000 Mons, Belgium

${ }^{3}$ Physics Laboratory, University of Athens, Solonos Str. 104, GR-10680 Athens, Greece

${ }^{4}$ Department of Physics, University of Bergen, Allégaten 55, N-5007 Bergen, Norway

${ }^{5}$ Dipartimento di Fisica, Università di Bologna and INFN, Via Irnerio 46, I-40126 Bologna, Italy

${ }^{6}$ Centro Brasileiro de Pesquisas Físicas, rua Xavier Sigaud 150, RJ-22290 Rio de Janeiro, Brazil and Depto. de Física, Pont. Univ. Católica, C.P. 38071 RJ-22453 Rio de Janeiro, Brazil and Inst. de Física, Univ. Estadual do Rio de Janeiro, rua São Francisco Xavier 524, Rio de Janeiro, Brazil ${ }^{7}$ Comenius University, Faculty of Mathematics and Physics, Mlynska Dolina, SK-84215 Bratislava, Slovakia

${ }^{8}$ Collège de France, Lab. de Physique Corpusculaire, IN2P3-CNRS, F-75231 Paris Cedex 05, France

${ }^{9} \mathrm{CERN}, \mathrm{CH}-1211$ Geneva 23, Switzerland

${ }^{10}$ Centre de Recherche Nucléaire, IN2P3 - CNRS/ULP - BP20, F-67037 Strasbourg Cedex, France

${ }^{11}$ Institute of Nuclear Physics, N.C.S.R. Demokritos, P.O. Box 60228, GR-15310 Athens, Greece

${ }^{12}$ FZU, Inst. of Physics of the C.A.S. High Energy Physics Division, Na Slovance 2, 180 40, Praha 8, Czech Republic

${ }^{13}$ Dipartimento di Fisica, Università di Genova and INFN, Via Dodecaneso 33, I-16146 Genova, Italy

${ }^{14}$ Institut des Sciences Nucléaires, IN2P3-CNRS, Université de Grenoble 1, F-38026 Grenoble Cedex, France

${ }^{15}$ Research Institute for High Energy Physics, SEFT, P.O. Box 9, FIN-00014 Helsinki, Finland

${ }^{16}$ Joint Institute for Nuclear Research, Dubna, Head Post Office, P.O. Box 79, 101000 Moscow, Russian Federation

${ }^{17}$ Institut für Experimentelle Kernphysik, Universität Karlsruhe, Postfach 6980, D-76128 Karlsruhe, Germany

${ }^{18}$ Institute of Nuclear Physics and University of Mining and Metalurgy, Ul. Kawiory 26a, PL-30055 Krakow, Poland

${ }^{19}$ Université de Paris-Sud, Lab. de l'Accélérateur Linéaire, IN2P3-CNRS, Bât. 200, F-91405 Orsay Cedex, France

${ }^{20}$ School of Physics and Materials, University of Lancaster, Lancaster LA1 4YB, UK

${ }^{21}$ LIP, IST, FCUL - Av. Elias Garcia, 14-1 ${ }^{\circ}$, P-1000 Lisboa Codex, Portugal

${ }^{22}$ Department of Physics, University of Liverpool, P.O. Box 147, Liverpool L69 3BX, UK

${ }^{23}$ LPNHE, IN2P3-CNRS, Universités Paris VI et VII, Tour 33 (RdC), 4 place Jussieu, F-75252 Paris Cedex 05, France

${ }^{24}$ Department of Physics, University of Lund, Sölvegatan 14, S-22363 Lund, Sweden

${ }^{25}$ Université Claude Bernard de Lyon, IPNL, IN2P3-CNRS, F-69622 Villeurbanne Cedex, France

${ }^{26}$ Universidad Complutense, Avda. Complutense s/n, E-28040 Madrid, Spain

${ }^{27}$ Univ. d'Aix - Marseille II - CPP, IN2P3-CNRS, F-13288 Marseille Cedex 09, France

${ }^{28}$ Dipartimento di Fisica, Università di Milano and INFN, Via Celoria 16, I-20133 Milan, Italy

${ }^{29}$ Niels Bohr Institute, Blegdamsvej 17, DK-2100 Copenhagen 0, Denmark

${ }^{30} \mathrm{NC}$, Nuclear Centre of MFF, Charles University, Areal MFF, V Holesovickach 2, 180 00, Praha 8, Czech Republic

${ }^{31}$ NIKHEF-H, Postbus 41882, NL-1009 DB Amsterdam, The Netherlands

${ }^{32}$ National Technical University, Physics Department, Zografou Campus, GR-15773 Athens, Greece

${ }^{33}$ Physics Department, University of Oslo, Blindern, N-1000 Oslo 3, Norway

${ }^{34}$ Dpto. Fisica, Univ. Oviedo, C/P. Pérez Casas, S/N-33006 Oviedo, Spain

${ }^{35}$ Department of Physics, University of Oxford, Keble Road, Oxford OX1 3RH, UK

${ }^{36}$ Dipartimento di Fisica, Università di Padova and INFN, Via Marzolo 8, I-35131 Padua, Italy

${ }^{37}$ Rutherford Appleton Laboratory, Chilton, Didcot OX11 OQX, UK

${ }^{38}$ Dipartimento di Fisica, Università di Roma II and INFN, Tor Vergata, I-00173 Rome, Italy

${ }^{39}$ Centre d'Etudes de Saclay, DSM/DAPNIA, F-91191 Gif-sur-Yvette Cedex, France

${ }^{40}$ Istituto Superiore di Sanità, Ist. Naz. di Fisica Nucl. (INFN), Viale Regina Elena 299, I-00161 Rome, Italy

${ }^{41}$ Instituto de Fisica de Cantabria (CSIC-UC), Avda. los Castros, S/N-39006 Santander, Spain, (CICYT-AEN93-0832)

${ }^{42}$ Inst. for High Energy Physics, Serpukov P.O. Box 35, Protvino, (Moscow Region), Russian Federation

${ }^{43} \mathrm{~J}$. Stefan Institute and Department of Physics, University of Ljubljana, Jamova 39, SI-61000 Ljubljana, Slovenia

${ }^{44}$ Fysikum, Stockholm University, Box 6730, S-113 85 Stockholm, Sweden

${ }^{45}$ Dipartimento di Fisica Sperimentale, Università di Torino and INFN, Via P. Giuria 1, I-10125 Turin, Italy

${ }^{46}$ Dipartimento di Fisica, Università di Trieste and INFN, Via A. Valerio 2, I-34127 Trieste, Italy and Istituto di Fisica, Università di Udine, I-33100 Udine, Italy

${ }^{47}$ Univ. Federal do Rio de Janeiro, C.P. 68528 Cidade Univ., Ilha do Fundão BR-21945-970 Rio de Janeiro, Brazil

${ }^{48}$ Department of Radiation Sciences, University of Uppsala, P.O. Box 535, S-751 21 Uppsala, Sweden

${ }^{49}$ IFIC, Valencia-CSIC, and D.F.A.M.N., U. de Valencia, Avda. Dr. Moliner 50, E-46100 Burjassot (Valencia), Spain

${ }^{50}$ Institut für Hochenergiephysik, Österr. Akad. d. Wissensch., Nikolsdorfergasse 18, A-1050 Vienna, Austria

${ }^{51}$ Inst. Nuclear Studies and University of Warsaw, Ul. Hoza 69, PL-00681 Warsaw, Poland

${ }^{52}$ Fachbereich Physik, University of Wuppertal, Postfach 100 127, D-42097 Wuppertal 1, Germany

${ }^{53}$ On leave of absence from IHEP Serpukhov
} 


\section{Introduction}

In the past all LEP experiments have published accurate measurements of the relative decay width of the $Z$ into B-hadrons, $R_{b}^{0}=\frac{\Gamma_{b \bar{b}}}{\Gamma_{\text {had }}}[1-5]$. Experimentally $R_{b}^{0}$ can be obtained with only very small corrections from the ratio of cross sections $R_{b}=\sigma\left(e^{+} e^{-} \rightarrow\right.$ $b \bar{b}) / \sigma\left(e^{+} e^{-} \rightarrow\right.$ hadrons $)$. The average value of $R_{b}^{0}[6]$ disagrees by about two standard deviations with the prediction of the Standard Model. To resolve the question of whether this deviation is real, new analyses including more data are needed. This paper presents three measurements of $R_{b}$ using data taken up to 1993 with the DELPHI detector at LEP.

The first analysis exploits the long lifetime of B-hadrons: $b$-quark hemispheres are tagged by the presence of large impact parameter tracks. Comparing single and double tag rates, $R_{b}$ can be measured together with the $b$-tagging efficiency. The second analysis uses the same tagging method as the first, however the tagging efficiency is obtained from hemispheres opposite to a high $p_{t}$ lepton. $R_{b}$ can then be measured from the single tag rate. Since the statistical precision is determined by the events having both an impact parameter tag and a lepton tag, the statistical correlation between the two methods is small. The systematic uncertainties are also largely different. Since the tagging method is rather simple, the tagging efficiency for light and $c$ quark events can be estimated reliably from simulation. In contrast, the third analysis uses a sophisticated tagging method combining thirteen vertex and event shape variables in a multivariate approach, so that all efficiencies can be estimated from data, using a complex least squares fit.

The two first methods have been published recently [3] using data from the 1991 and 1992 running periods, and are updated here using 1993 data. The third one, using the 1992 and 1993 data, updates the 1991 result [7]. The three analyses are combined, taking into account correlations.

\section{The DELPHI Detector}

The DELPHI detector and its performance have been described in detail in ref. $[8,9]$. Only the details most relevant to this analysis are mentioned here.

In the barrel region, the charged particle tracks are measured by a set of cylindrical tracking detectors whose axes are parallel to the $1.2 \mathrm{~T}$ solenoidal magnetic field and to the beam direction. The time projection chamber (TPC) is the main tracking device. The TPC is a cylinder with a length of $3 \mathrm{~m}$, an inner radius of $30 \mathrm{~cm}$ and an outer radius of $122 \mathrm{~cm}$. Between polar angles, $\theta$, of $39^{\circ}$ and $141^{\circ}$ with respect to the beam direction, tracks are reconstructed using up to 16 space points. Outside this region $\left(21^{\circ}\right.$ to $39^{\circ}$ and $141^{\circ}$ to $\left.159^{\circ}\right)$, tracks can be reconstructed using at least 4 space points.

Additional precise $R \Phi$ measurements, in the plane perpendicular to the magnetic field, are provided at larger and smaller radii by the Outer and Inner detectors respectively. The Outer Detector (OD) has five layers of drift cells at radii between 198 and $206 \mathrm{~cm}$ and covers polar angles from $42^{\circ}$ to $138^{\circ}$. The Inner Detector (ID) is a cylindrical drift chamber having inner radius of $12 \mathrm{~cm}$ and outer radius of $28 \mathrm{~cm}$. It covers polar angles between $29^{\circ}$ and $151^{\circ}$. It contains a jet chamber section providing $24 R \Phi$ coordinates, surrounded by five layers of proportional chambers giving both $R \Phi$ and longitudinal $z$ coordinates.

The micro-vertex detector (VD) is located between the LEP beam pipe and the ID [10]. It consists of three concentric layers of silicon microstrip detectors at radii of $6.3,9$ and

$11 \mathrm{~cm}$ from the beam line. For all layers the microstrip detectors provide hits in the 
$R \Phi$-plane with a measured intrinsic resolution of about $8 \mu \mathrm{m}$. The polar angle coverage for charged particles hitting all three layers of the detector is $42.5^{\circ}$ to $137.5^{\circ}$.

The barrel electromagnetic calorimeter (HPC) covers polar angles between $42^{\circ}$ and $138^{\circ}$. It is a gas-sampling device which provides complete three-dimensional charge information in the same way as a time projection chamber. Each shower is sampled nine times in its longitudinal development. Along the drift direction, parallel to the DELPHI magnetic field, the shower is sampled every $3.5 \mathrm{~mm}$; in the plane perpendicular to the drift the charge is collected by cathode pads of variable size, ranging from $2.3 \mathrm{~cm}$ in the inner part of the detector to $7 \mathrm{~cm}$ in the outer layers. The excellent granularity allows good separation between close particles in three dimensions and hence good electron identification even inside jets.

In the forward region the tracking is complemented by two sets of planar drift chambers (FCA and $\mathrm{FCB}$ ), at distances of $\pm 165 \mathrm{~cm}$ and $\pm 275 \mathrm{~cm}$ from the interaction point. A lead glass calorimeter (EMF) is used to reconstruct electromagnetic energy in the forward region.

Muon identification in the barrel region is based on a set of muon chambers (MUB), covering polar angles between $53^{\circ}$ and $127^{\circ}$. It consists of six active cylinders of drift chambers, two inside the return yoke of the magnet after $90 \mathrm{~cm}$ of iron (inner layer) and four outside after a further $20 \mathrm{~cm}$ of iron (outer and peripheral layers). The inner and outer modules have similar azimuthal coverage. The gaps in azimuth between adjacent modules are covered by the peripheral modules. Therefore a muon traverses typically either two inner layer chambers and two outer layer chambers, or just two peripheral layer chambers. Each chamber measures the $R \Phi$ coordinate to $2-3 \mathrm{~mm}$. Measuring $R \Phi$ in both the inner layer and the outer or peripheral layer determines the azimuthal angle of muon candidates leaving the return yoke within about $\pm 1^{\circ}$. These errors are much smaller than the effects of multiple scattering on muons traversing the iron.

In the forward region the muon identification is done using two sets of planar drift chambers (MUF) covering the angular region between $11^{\circ}$ and $45^{\circ}$. The first set is placed behind $85 \mathrm{~cm}$ of iron and the second one behind an additional $20 \mathrm{~cm}$. Each set consists of two orthogonal layers of drift chambers where the anode is read out directly and the cathode via a delay line to measure the coordinate along the wire. The resolution in both coordinates is about $4 \mathrm{~mm}$.

\section{Event Selection}

The criteria to select charged tracks and to identify hadronic $Z$ decays were identical to those described in [3]. Charged particles were accepted if:

- their polar angle was between $20^{\circ}$ and $160^{\circ}$,

- their track length was larger than $30 \mathrm{~cm}$,

- their impact parameter relative to the interaction point was less than $2.5 \mathrm{~cm}$ in the plane perpendicular to the beam direction and less than $10 \mathrm{~cm}$ along the beam direction,

- their momentum was larger than $200 \mathrm{MeV} / \mathrm{c}$ with relative error less than $100 \%$. 
Neutral particles detected in the HPC were required to have measured energy larger than $700 \mathrm{MeV}$, those detected in the EMF larger than $400 \mathrm{MeV}$.

Events have been selected by requiring:

- at least 7 reconstructed charged particles,

- the summed energy of the charged particles had to be larger than $15 \%$ of the centre of mass energy, and at least $3 \%$ of it in each of the forward and backward hemispheres with respect to the beam axis.

The efficiency to find hadronic $Z$ decays with these cuts was about $95 \%$ with only very small bias towards a specific flavour, and all backgrounds were below $0.1 \%$.

About 700000 hadronic $Z$ decays have been selected from each of the 1993 and 1992 data samples, where the exact numbers vary slightly for the different analyses due to different requirements on the detector availability. The ratio of the cross section $Z \rightarrow b \bar{b}$ to the total hadronic cross section varies very little at centre of mass energies close to the $Z$ mass. Thus no selection on the centre of mass energy has been made. However the validity of this assumption has been tested in sections 4.4 and 6.2. A sample about twice the data statistics of $Z \rightarrow q \bar{q}$ events has been simulated using the Lund parton shower Monte Carlo JETSET 7.3 [11] (with parameters optimized by DELPHI) and the DELPHI detector simulation [9]. In addition dedicated samples of $Z \rightarrow b \bar{b}$ events have been generated. The simulated events have been passed through the same analysis chain as the real ones.

\section{The Impact Parameter Analysis}

The method used for this measurement of $R_{b}$ is nearly identical to the one described in [3]. The basic feature of the method is the extraction of $R_{b}$ from the comparison of the single and double tag rates:

$$
\begin{aligned}
R_{H} & =R_{b} \cdot \epsilon_{b}+R_{c} \cdot \epsilon_{c}+\left(1-R_{b}-R_{c}\right) \cdot \epsilon_{u d s}, \\
R_{E} & =R_{b} \cdot\left\{\epsilon_{b}^{2}+\rho_{b} \cdot\left(\epsilon_{b}-\epsilon_{b}^{2}\right)\right\}+R_{c} \cdot \epsilon_{c}^{2}+\left(1-R_{b}-R_{c}\right) \cdot \epsilon_{u d s}^{2},
\end{aligned}
$$

where $R_{H}$ is the fraction of tagged hemispheres and $R_{E}$ the fraction of events in which both hemispheres are tagged. $\epsilon_{q}$ is the efficiency to tag a hemisphere originating from a primary quark $q(q=b, c, u d s)$. The factor $\rho_{b}=\frac{\epsilon_{b}^{(d)}-\epsilon_{b}^{2}}{\epsilon_{b}\left(1-\epsilon_{b}\right)}$ accounts for hemisphere correlations in the tagging efficiencies for $b$ quarks, where $\epsilon_{b}^{(d)}$ is the double tag efficiency for $b$ events. For the other quark species, these correlations can safely be neglected. If $\rho_{b}, \epsilon_{u d s}$ and $\epsilon_{c}$ are calculated from the simulation and $R_{c}$ is imposed from other measurements or from the Standard Model, $R_{b}$ and $\epsilon_{b}$ can be measured simultaneously from the data. Thus a good knowledge of the details of B-hadron decays is not needed.

As the $b$-tagging variable, the probability $P_{H}$ that all tracks from a hemisphere originate from a common primary vertex was used; this is identical to the tagging variable described in [3]. Where to cut in $P_{H}$ is arbitrary; for the results presented here, the cut that minimizes the total error has been chosen.

In the following, only the features different from the 1992 analysis will be described in detail. Since for the measurement of impact parameters the VD is essential, the method is limited to events with most tracks inside the VD acceptance. For this reason a cut on $\left|\cos \theta_{\text {thrust }}\right|<0.65$ is applied. This cut is harder than the one applied in [3], because 
in 1993, due to some inconsistency in the VD position between data and simulation, the description of the edge of the VD acceptance was slightly inaccurate.

Since for this analysis a good description of the data by the simulation for udsc quarks is required, some tuning of the impact parameter distribution in the simulation has to be performed. This procedure has been refined with respect to the 1992 analysis, basically by taking into account small inhomogeneities in the azimuthal angle [12]. This leads to substantially smaller uncertainties due to the understanding of the detector resolution.

\subsection{Estimates of Efficiencies and Correlations}

The analysis was performed at many different cut values. The total error was found to be almost constant for cuts between $\log _{10} P_{H}<-2.7$ and $\log _{10} P_{H}<-2.9$. For harder cuts, the contribution of the statistical error to the total error is larger. Therefore the hardest of these cuts was chosen, to decrease the correlation between this analysis and the others.

With this cut, the values of the non- $b$ efficiencies $\left(\epsilon_{c}, \epsilon_{u d s}\right)$ and the hemisphere correlation $\left(\rho_{b}\right)$ were extracted from the simulation, and the possible sources of uncertainties were included as systematic errors. In many cases, input parameters have to be chosen from measurements not made at LEP (branching ratios, lifetimes etc.). The LEP experiments have agreed on a common set of all these parameters, and on a procedure to evaluate the errors arising from them [6]. In all cases these recommendations have been followed.

The values of the efficiencies were found to be:

$$
\begin{aligned}
\epsilon_{u d s} & =(0.260 \pm 0.013) \times 10^{-2}, \\
\epsilon_{c} & =(1.67 \pm 0.15) \times 10^{-2} .
\end{aligned}
$$

\begin{tabular}{|c|c|c|}
\hline Source of systematics & Range & $\Delta \epsilon_{u d s} \times 10^{4}$ \\
\hline MC statistics & & \pm 0.5 \\
\hline Detector resolution & & \pm 0.8 \\
\hline$K^{0}$ & Tuned JETSET $\pm 10 \%$ & \pm 0.4 \\
\hline Hyperons & Tuned JETSET $\pm 10 \%$ & \pm 0.1 \\
\hline Photon conversions & $\pm 30 \%$ & \pm 0.1 \\
\hline Gluon splitting $g \rightarrow b \bar{b}$ & $(0.16 \pm 0.08) \%$ & \pm 0.7 \\
\hline Gluon splitting $g \rightarrow c \bar{c}$ & $(1.5 \pm 0.8) \%$ & \pm 0.3 \\
\hline Total & & \pm 1.3 \\
\hline
\end{tabular}

The different sources of systematic uncertainties are given in tables 1 and 2 .

Table 1: Systematic errors of light quark efficiency $\epsilon_{u d s}$.

Light quark events are tagged mainly because of statistical fluctuations, which are present in the same way for positive and negative impact parameters. The systematic error on $\epsilon_{u d s}$ coming from the differences in resolution between data and simulation has therefore been estimated as the difference of the tagging efficiencies in data and in simulation when the hemisphere probabilities were computed using tracks with negative impact parameters.

Charm events, on the contrary, are tagged because charmed particles have detectable lifetimes. The effect of the detector resolution on $\epsilon_{c}$ can be estimated by using in the 


\begin{tabular}{||l|c|c||}
\hline Source of systematics & Range & $\Delta \epsilon_{c} \times 10^{4}$ \\
\hline MC statistics & & \pm 2.0 \\
Detector resolution & $0.557 \pm 0.053$ & \pm 4.0 \\
$D^{0}$ fraction in $c \bar{c}$ events & \pm 0.6 \\
$D^{+}$fraction in $\bar{c}$ events & $0.248 \pm 0.037$ & \pm 9.3 \\
$\left(D^{0}+D^{+}\right)$fraction in $c \bar{c}$ events & $0.80 \pm 0.07$ & \pm 5.0 \\
$D_{s}$ fraction in $c \bar{c}$ events & $0.12 \pm 0.05$ & \pm 4.3 \\
$D_{\text {decay multiplicity }}$ & $2.53 \pm 0.06$ & \pm 3.8 \\
$B R\left(D \rightarrow K^{0} X\right)$ & $0.46 \pm 0.06$ & \pm 6.5 \\
$D^{0}$ lifetime & $0.420 \pm 0.008 \mathrm{ps}$ & \pm 1.4 \\
$D^{+}$lifetime & $1.066 \pm 0.023 \mathrm{ps}$ & \pm 1.9 \\
$D_{s}$ lifetime & $0.450_{-0.026}^{+0.030} \mathrm{ps}$ & \pm 1.4 \\
$\Lambda_{c}$ lifetime & $0.191_{-0.012}^{+0.015} \mathrm{ps}$ & \pm 0.0 \\
$\left\langle x_{E}(c)\right\rangle$ & $0.49 \pm 0.02$ & \pm 3.0 \\
\hline Total & \multicolumn{2}{|c||}{} \\
\hline
\end{tabular}

Table 2: Systematic errors of charm quark efficiency $\epsilon_{c}$

simulation the calibration curve obtained from the data. Since the assignment of errors to the impact parameters is the same in data and simulation, the difference in the resolution curve reflects the difference in the true resolution. All other sources of systematic error have been estimated as suggested in [6].

The correlation between hemispheres in $b$ events was evaluated from the simulation to be

$$
\left.\rho_{b}=(-1.28 \pm 0.13(\text { stat. }) \pm 0.09 \text { (syst. })\right) \times 10^{-2}
$$

where the systematic error was evaluated as follows. The correlation can be described mainly in terms of four sources:

- radiation of hard gluons: this source acts in two ways. Due to gluon radiation, energy is taken away from the B-hadrons. Since the resolution is largely determined by the multiple scattering in the beam pipe, this lowers the tagging efficiency, which leads to a positive correlation. In about $2 \%$ of the cases both $\mathrm{B}$-hadrons are boosted into the same hemisphere, leading to a negative correlation.

- the polar angle of the thrust axis: as the polar angle is varied, both jets tend to be in a region of either good or less good VD acceptance simultaneously, which leads to a positive correlation. Since a much harder cut was imposed on the polar angle of the thrust axis in this analysis than in [3], this source was reduced to a negligible level.

- the azimuthal angle of the jets: due to dead or noisy modules in the vertex detector, the efficiency was not completely independent of $\Phi$. However in the data sample presented here, most modules were highly efficient.

- biases of the fitted production vertex relative to the true $Z$ decay point due to the inclusion of tracks from $b$ decays lead to a negative correlation.

Figure 1 shows the total correlation as a function of the cut value, together with each of these four components and their sum. In the region that is used for the analysis, the total correlation is well described by the sum of the components listed above.

To obtain the systematic error on the correlation estimate from the simulation, the fraction of tagged events was measured as a function of the relevant variable in data and 


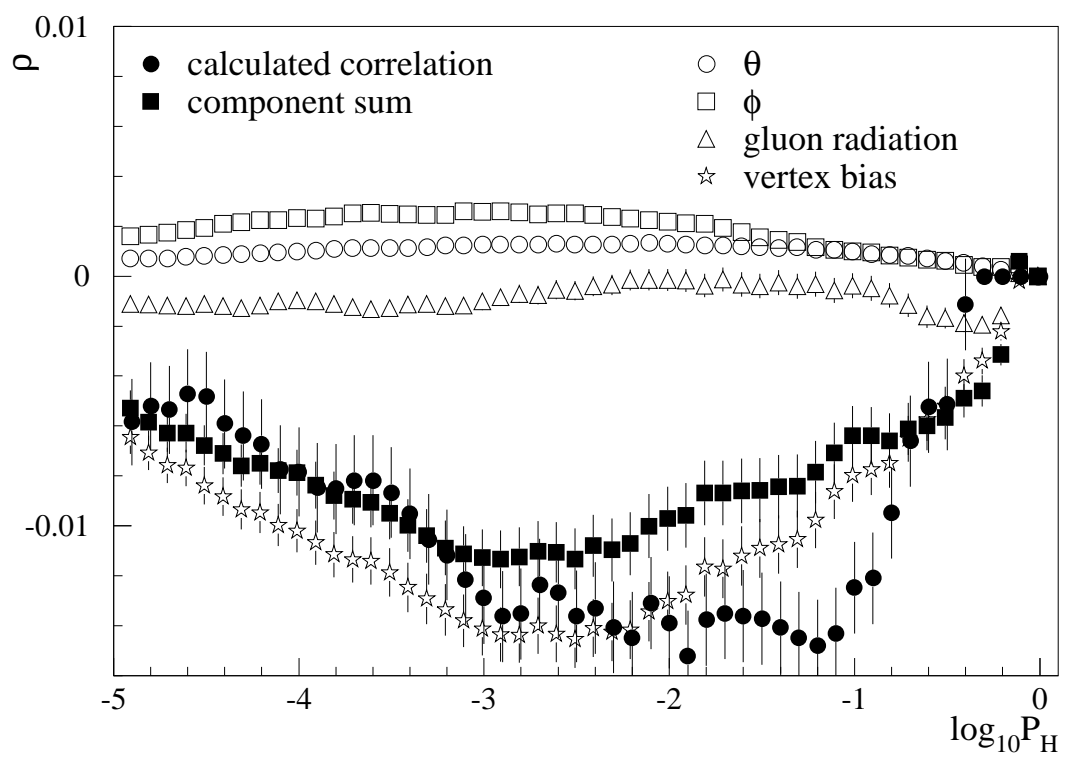

Figure 1: Total hemisphere correlation and individual contributions as a function of the cut value $\log _{10} P_{H}$.

in simulation using all events. From this, the correlation due to that single variable was calculated. The result was scaled by the ratio of the correlations in $b \bar{b}$ events and in all events obtained from the simulation. The larger of either a) the difference between the data and simulation measurement, or b) the statistical error on this difference, was taken as the error estimate. However, for $\log _{10} P_{H}<-2.9$ the statistical error was always larger than the difference.

In the case of gluon radiation, thrust was used as the testing variable. To account partly for the cancellation of the two different effects, the thrust was signed in each hemisphere to be positive in the hemisphere with the larger invariant mass and negative in the other one. To verify that this procedure gives a reliable estimate of the uncertainty due to gluon radiation, several tests were made with the Monte Carlo. Events were rejected if they had a) both $b$ 's in one hemisphere, b) a thrust value less than 0.96 or c) more than 3 jets. In each case, the total hemisphere correlation and the single source correlation from thrust were calculated, and compared with the values obtained from all events. In all cases, the change in the single source correlation obtained from thrust was larger than the change in the total hemisphere correlation. Thus all simulation problems of these types would have been detected by the comparison made between data and simulation. Figure 2 shows the correlation obtained from the signed thrust, when tagging on the opposite hemisphere to reduce the distortions from light quark events. It should be noted that the agreement between data and simulation is not affected either by tagging or not tagging on the opposite hemisphere, or by using the signed or the normal thrust. As an additional cross-check, the hemisphere correlation was calculated using 1 million hadronic $Z$ decays generated with the matrix element option of the JETSET Monte Carlo program [11]. Within the statistical errors, the same correlation was found. 
To estimate the correlation due to the vertex bias, a primary vertex was found separately for each hemisphere of each event, using the beam spot information and the tracks in the hemisphere that had been used to reconstruct the common primary vertex. The correlation was calculated as function of the distance between the two hemisphere vertices. This distance was signed by comparing how far downstream the vertex moved when the beam spot was removed from the vertex fit: it was positive in the hemisphere where the movement downstream was larger and negative in the other one. Since this distance is itself an efficient $b$-tagging variable, the correlation observed is strongly affected by the presence of light quark events. Therefore a hemisphere was used to measure the vertex bias correlation only if the opposite hemisphere was tagged as a $b$ hemisphere. The correlations extracted from data and simulation are shown in figure 3 . The correlation obtained by this method is not exactly equal to the one labelled "vertex bias" in figure 1. However, since it is used only to extract a systematic error, small distortions of this distribution with respect to the true "vertex bias" correlation are not important.

The different sources of systematic error on $\rho_{b}$ are listed in table 3 .

\begin{tabular}{||c|c||}
\hline Source of systematics & $\Delta \rho_{b} \times 10^{4}$ \\
\hline Resolution function & \pm 1.0 \\
Polar angle acceptance & \pm 2.2 \\
Azimuthal angle acceptance & \pm 3.9 \\
Hard gluon emission & \pm 6.3 \\
Vertex bias & \pm 4.8 \\
\hline total & \pm 9.2 \\
\hline
\end{tabular}

Table 3: Systematic errors on the correlation factor $\rho_{b}$.

\subsection{Results}

700000 hadronic $Z$ decays were selected, of which 413671 passed the $\left|\cos \theta_{\text {thrust }}\right|$ cut. Of these, 42537 single hemispheres were tagged and 3930 events were double tagged for $\log _{10} P_{H}<-2.9$. The bias towards $b$ events in the event selection was found to be very small, $(0.79 \pm 0.31) \cdot 10^{-3}$, and was corrected for. Using the above values of the efficiencies and the correlation, with their errors, the measured value of $R_{b}$ is:

$$
\left.R_{b}=0.2218 \pm 0.0029(\text { stat. }) \pm 0.0029 \text { (syst. }\right)-0.022 \frac{R_{c}-0.172}{0.172} .
$$

The $b$ hemisphere tagging efficiency was found to be $\epsilon_{b}=0.210 \pm 0.003$, compared to $\epsilon_{b}(M C)=0.209$ obtained from the simulation. The breakdown of the error for the given cut on $P_{H}$ is given in table 4.

As a cross-check of this measurement, the comparison of $R_{b}$ values for different tagging cuts is given in figure 4 . The measured value of $R_{b}$ is stable over a wide range of variation of the cut value and therefore of the efficiencies and of the correlation.

\subsection{Combination with the 91/92 Analysis}

In order to combine the analysis presented here with a similar one published in [3], the following assumptions were made. 


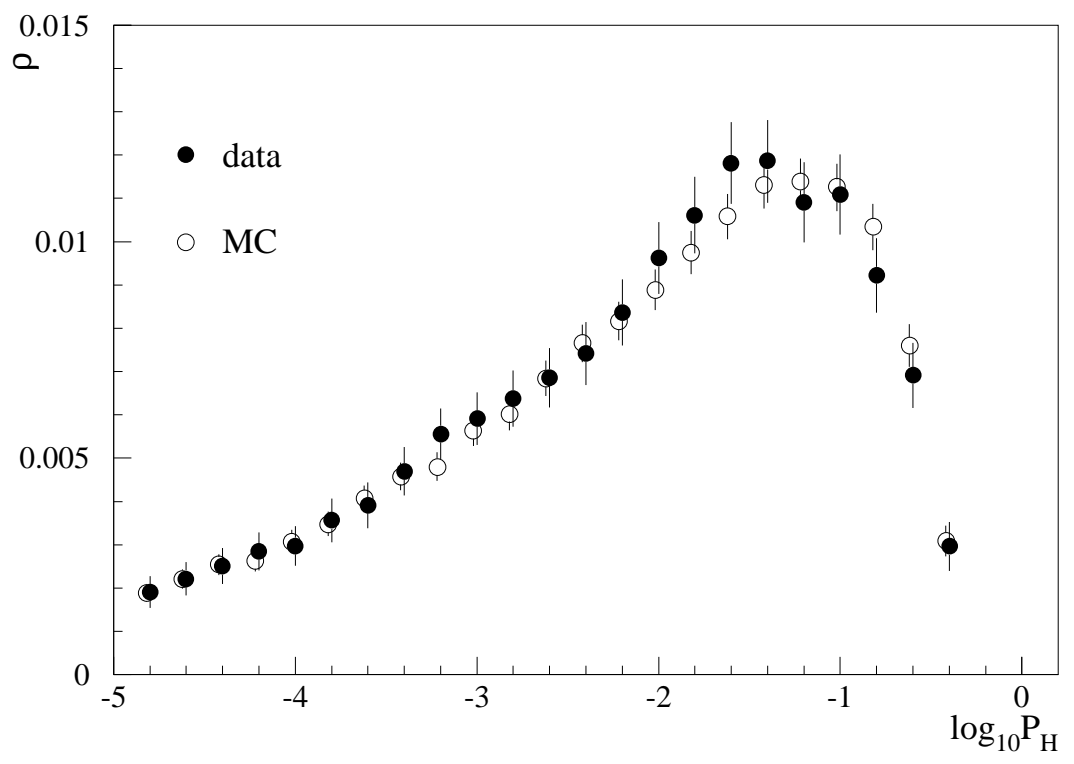

Figure 2: Hemisphere correlation due to gluon radiation. The correlation obtained from the signed thrust is shown.

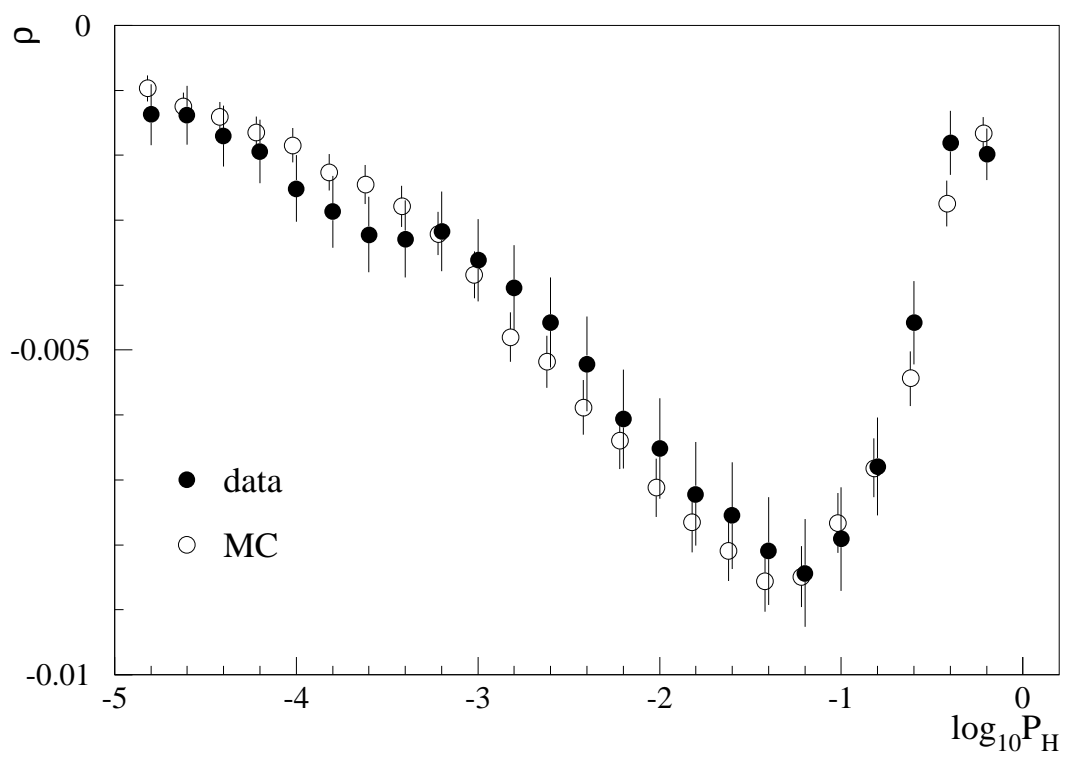

Figure 3: Hemisphere correlation due to vertex bias. The closed and open points show (for data and simulation respectively) the values obtained with the procedure described at the end of section 4.1. 


\begin{tabular}{||c|c||}
\hline Error Source & $\Delta R_{b} \times 10^{3}$ \\
\hline Statistical error & \pm 2.9 \\
Light quark efficiency & \pm 0.8 \\
Charm efficiency & \pm 2.4 \\
Correlation & \pm 1.5 \\
\hline Total & \pm 4.1 \\
\hline
\end{tabular}

Table 4: Sources of errors for the measurement of $R_{b}$.

- All statistical errors were assumed to be independent.

- The errors in the hemisphere correlations due to hard gluon emission were assumed to be fully correlated between the two results, as were those for the polar angle acceptance. The uncertainty in the vertex bias is strongly connected with the uncertainty in the B-hadron lifetime. Therefore the vertex bias error in this analysis was assumed to be fully correlated with the error labelled "B-hadron lifetime" in [3]. The errors related to azimuthal dependences were assumed to be uncorrelated, since the dependences arise mainly from dead VD modules, which are repaired year by year.

- The tuning of the resolution function in the simulation is done year by year by comparing the simulation with the data, so the errors due to the uncertainties in the resolution functions were also assumed to be independent.

- The errors due to the modelling of the light and charm quarks were assumed to be fully correlated.

With these assumptions, the result for the combined 1991/92/93 data is:

$$
R_{b}=0.2219 \pm 0.0018(\text { stat. }) \pm 0.0028(\text { syst. })-0.022 \frac{R_{c}-0.172}{0.172} \text {. }
$$

\subsection{Energy dependence}

In 1993, data were taken at three different centre of mass energies $(\sqrt{s}=$ 89.49, 91.25, $93.08 \mathrm{GeV})$. As photon exchange and $\gamma-Z$ interference are strongly suppressed at energies close to the $Z$ resonance, $R_{b}(\sqrt{s})$ is predicted to be almost constant in the Standard Model. However, if $R_{b}$ is affected by the interference of the $Z$ with a $Z^{\prime}$ almost degenerate in mass, as recently suggested by Caravaglios and Ross [13], some energy dependence can be expected if the mass and width of the $Z^{\prime}$ are not exactly equal to those of the $Z$. Since the $b$-tagging efficiency varies only very little within the energy range considered here, no complicated single to double tag comparison is needed to measure $\frac{R_{b}(\sqrt{s})}{R_{b}(91.25 \mathrm{GeV})}$. Instead, simply the ratio of the fraction of tagged events can be used, with small corrections due to changes in the $b$-tagging efficiency and almost negligible corrections due to background. These corrections were calculated using the Monte Carlo simulation. The measurement was performed using event probabilities instead of hemisphere probabilities. Several different values of the event probability cut were used, and a minimum statistical error was found at $\log _{10} P_{E}<-1.6$. At this value of the cut, the $b$-tagging efficiency varied by a relative amount of $\pm 0.5 \%$ with respect to that at the $Z$ peak and was about $70 \%$, while the efficiency to tag $c(u d s)$ events was about $20 \%(4 \%)$. To avoid any systematic uncertainties due to time dependence of the $b$-tagging efficiency, the data taken in the first part of the year, where LEP ran only at $\sqrt{(s)}=91.25 \mathrm{GeV}$, 


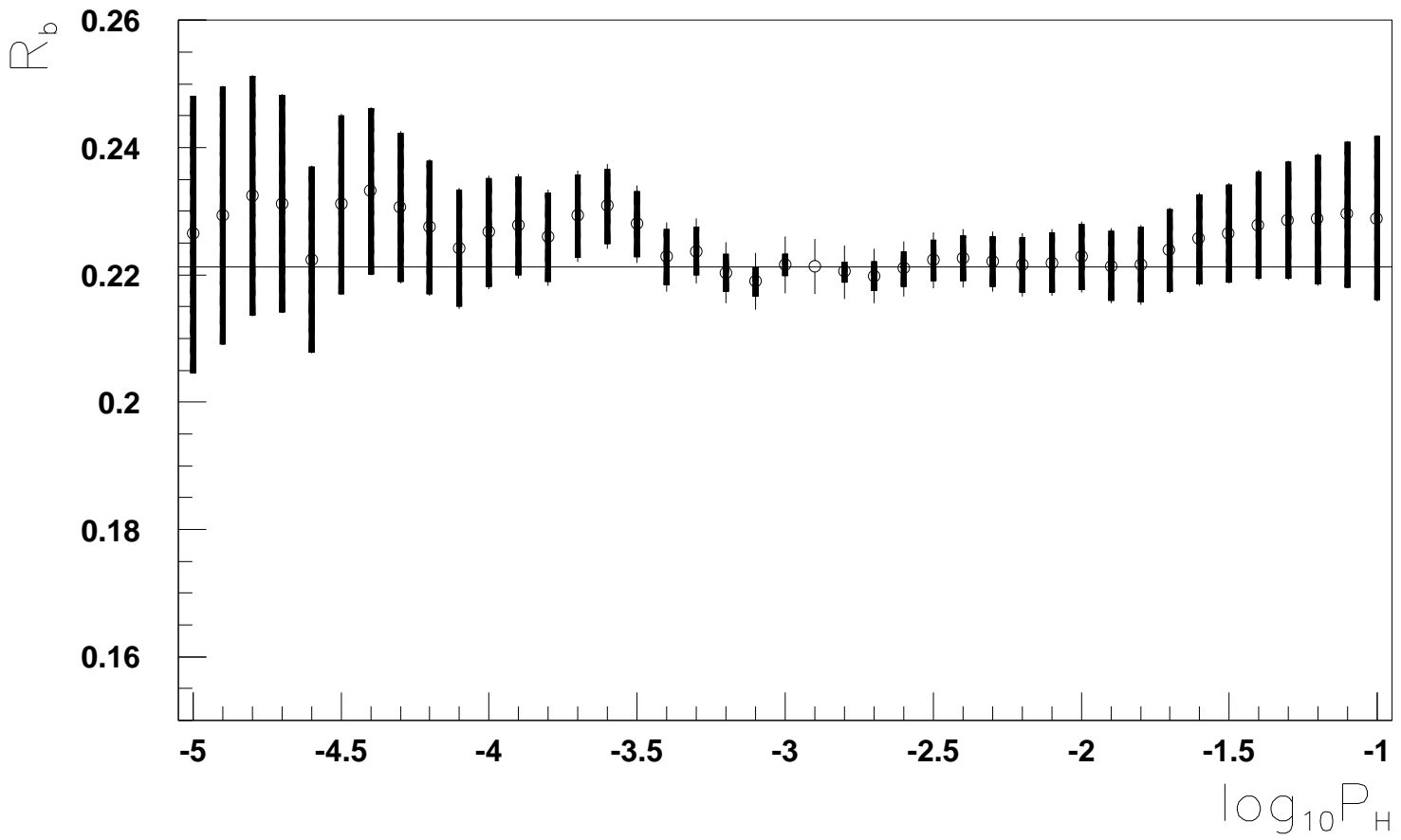

Figure 4: The value of $R_{b}$ with its total error as a function of the cut on $\log _{10} P_{H}$. The horizontal line corresponds to the value measured at the reference point, $\log _{10} P_{H}<-2.9$. The thin error bar corresponds to the total error, the thick one indicates the error on the change in $R_{b}$ from the value at the reference point.

on the $Z$ peak, were neglected. With these requirements the following ratios were found:

$$
\begin{aligned}
& R_{-}=\frac{R_{b}(89.49 \mathrm{GeV})}{R_{b}(91.25 \mathrm{GeV})}=0.982 \pm 0.015, \\
& R_{+}=\frac{R_{b}(93.08 \mathrm{GeV})}{R_{b}(91.25 \mathrm{GeV})}=0.997 \pm 0.016 .
\end{aligned}
$$

The error is statistical only, including the limited Monte Carlo statistics at the off-peak points. All systematic uncertainties were found to be negligible. The Standard Model predicts a ratio of $0.997(0.998)$ for $R_{-}\left(R_{+}\right)$. Figure 5 shows the stability of the measurement with respect to the cut value. Figure 6 compares the result with the Standard Model prediction.

\section{Mixed tag Analysis}

This analysis used the impact parameter $b$-tagging technique described in section 4 , but the efficiency of the tag was measured using a sample of events enriched in semileptonic $b$ decays. The track and event selections, including the polar angle cut, were basically identical to the ones used in section 4 .

The lepton identification methods are described in [3]. To increase the fraction of $b$ events in the sample, a cut was applied to the transverse momentum of the lepton with respect to the axis of the jet, after removal of the lepton itself $\left(p_{t}^{\text {out }}\right)$. The cut $p_{t}^{\text {out }}>1.5 \mathrm{GeV} / \mathrm{c}$ was used. If $\epsilon_{q}$ is the probability of tagging one hemisphere using the impact parameter technique when a flavour $q$ is produced in the $Z$ decay, and $P_{q}$ is the 


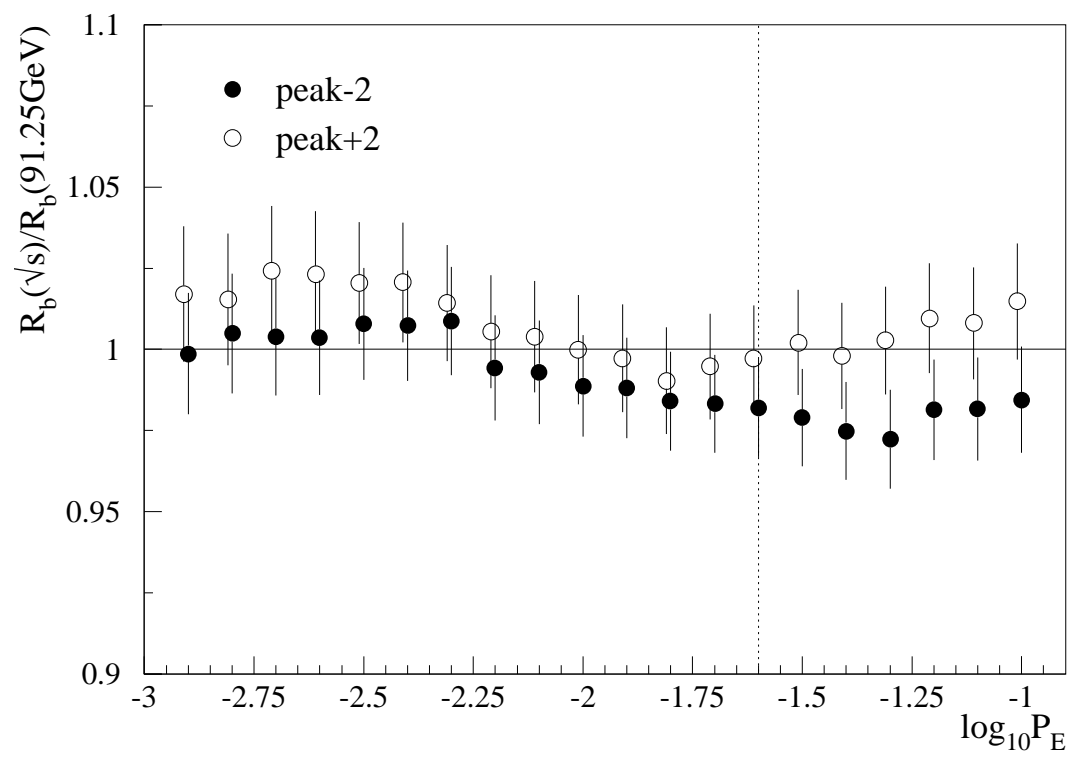

Figure 5: Ratio of the off-peak and on-peak $R_{b}$ values as a function of the cut value. The vertical dotted line marks the cut used for the central values.

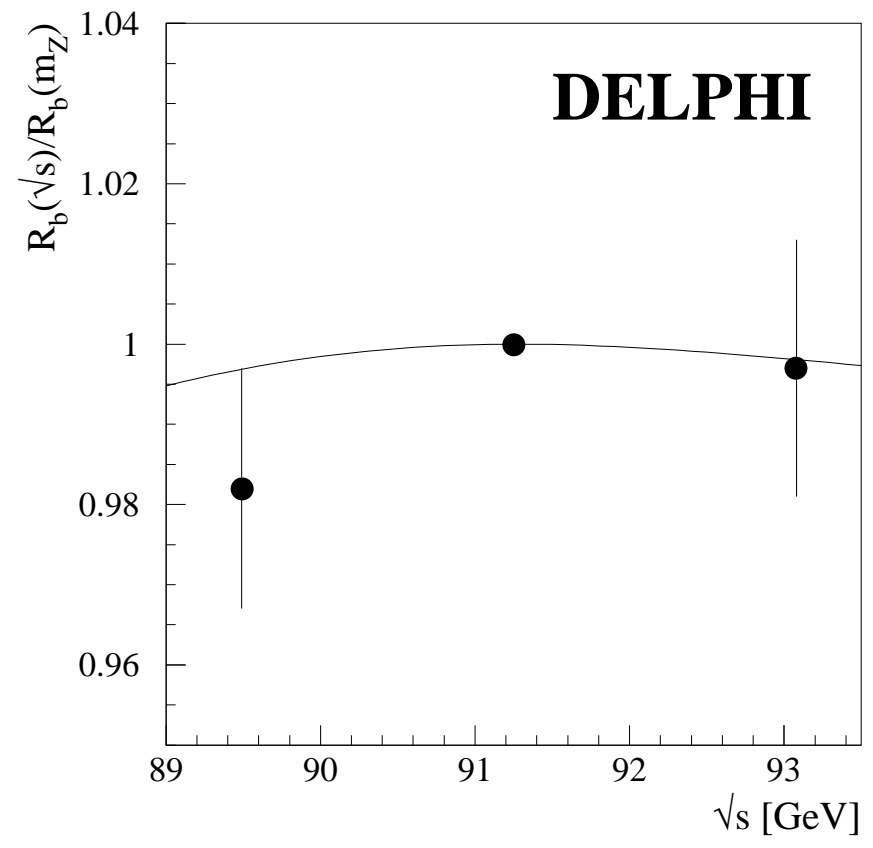

Figure 6: Ratio of the off-peak and on-peak $R_{b}$ values as a function of $\sqrt{s}$. The solid line shows the Standard Model prediction. 
fraction of events from the flavour $q$ in the lepton subsample, then

$$
\begin{aligned}
& f_{1}=\epsilon_{b} R_{b}+\epsilon_{c} R_{c}+\epsilon_{u d s} R_{u d s}, \\
& f_{2}=c_{l}^{b} \epsilon_{b} P_{b}+c_{l}^{c} \epsilon_{c} P_{c}+c_{l}^{u d s} \epsilon_{u d s} P_{u d s},
\end{aligned}
$$

where $f_{1}$ is the fraction of hemispheres in hadronic $Z$ events tagged by the impact parameter selection, and $f_{2}$ is the fraction of semileptonic decays tagged by the impact parameter selection in the hemisphere opposite to the lepton. To extract the efficiency of the lifetime tags with adequate precision, accurate knowledge of the flavour composition of the lepton sample, as expressed by the coefficients $P_{q}$, is required. Section 5.1 is devoted to this topic.

The coefficients $c_{l}^{q}$ account for the correlations between the impact parameter and lepton tags in opposite hemispheres, and were computed by simulation. Due to the smallness of the contamination from $c$ and light quarks, only the knowledge of $c_{l}^{b}$ was relevant for the measurement. With the requirement $\log _{10} P_{H}<-2.5$, the efficiencies for tagging the different flavours and the correlation coefficient $c_{l}^{b}$ were estimated in the simulation as:

$$
\begin{aligned}
\epsilon_{u d s} & =(0.71 \pm 0.01) \% \\
\epsilon_{c} & =(3.63 \pm 0.04) \% \\
c_{l}^{b} & =1.014 \pm 0.008 \pm 0.005
\end{aligned}
$$

The systematic uncertainty on the correlation coefficient $c_{l}^{b}$ was determined in the same way as that on the correlation $\rho_{b}$ in the impact parameter analysis.

The total data sample after all cuts consisted of 426786 events, in which 67717 hemispheres were tagged with the above probability cut. 11204 events with a high $p_{t}$ lepton were found, 2891 of which were also $b$-tagged. From these numbers the values:

$$
\begin{aligned}
\epsilon_{b}^{\text {data }} & =(30.63 \pm 0.51) \%, \\
R_{b} & =0.2240 \pm 0.0039
\end{aligned}
$$

were derived, where the errors are only statistical. The value of $\epsilon_{b}$ given by the simulation was $(30.30 \pm 0.05) \%$. As the cut on the lepton $p_{t}^{\text {out }}$ is an arbitrary parameter, chosen to minimize the total error, the variation of the $R_{b}$ value when changing the $p_{t}^{\text {out }}$ selection was checked. Figure 7 shows the result of this test.

The systematic errors will be discussed in section 5.2.

\subsection{The Composition of the Lepton Sample}

A fit to the single and di-lepton distributions (performed on the 1993 data sample by the method discussed in ref. [3] ) allowed a precise determination of the fractions $P_{b}$ and $P_{c}$ of events from $b$ and $c$ quarks in the lepton sample. These fractions (called "purities" below) were computed as a function of $p_{t}^{\text {out }}$, in the subset of hadronic events selected for the impact parameter analysis. The most energetic candidate was used when more than one lepton was found in the event (due to the high $p_{t}^{\text {out }}$ cut, this applied to less than $1 \%$ of the cases). The requirement $p_{t}^{\text {out }}>1.5 \mathrm{GeV} / \mathrm{c}$ was applied in order to minimize the overall error on $R_{b}$. The purities of the sample were estimated as:

$$
\begin{aligned}
& P_{b}=(81.17 \pm 0.79) \% \\
& P_{c}=(9.56 \pm 0.76) \%
\end{aligned}
$$




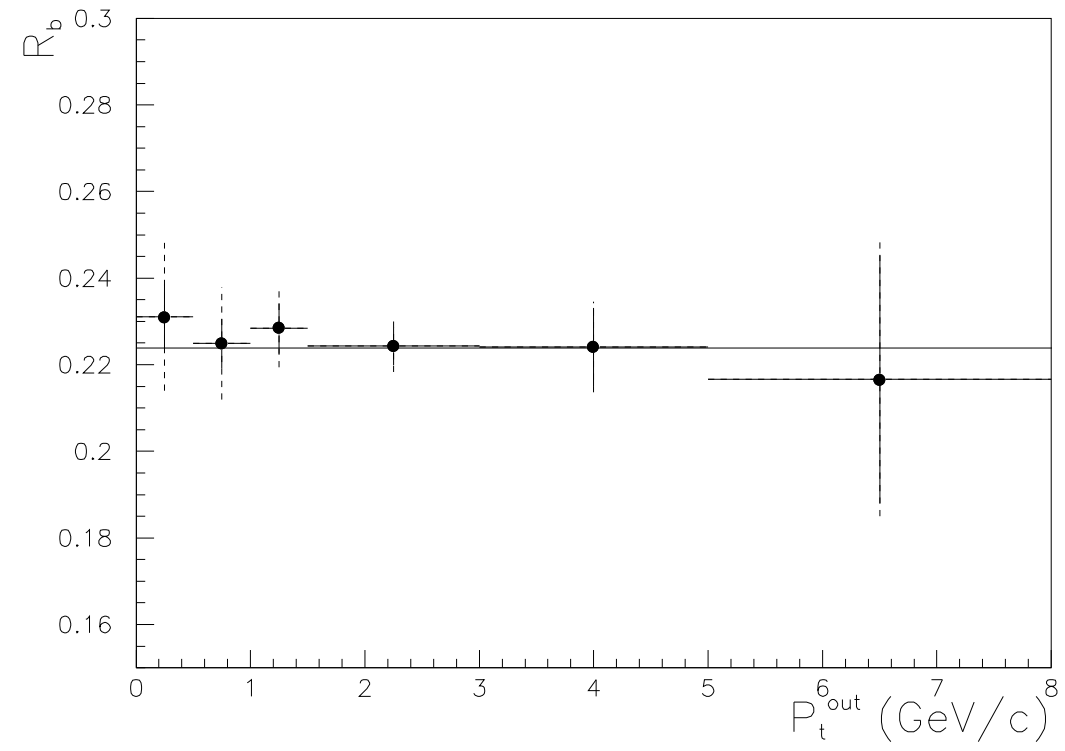

Figure 7: $R_{b}$ versus the transverse momentum of the lepton. The bins are uncorrelated. The solid error bars indicate the statistical errors and the dashed ones the total errors. The horizontal line shows the result quoted in the text, obtained with the cut $p_{t}^{\text {out }}>1.5 \mathrm{GeV} / \mathrm{c}$.

Table 5 shows the contributions to the total error on $P_{b}$. $P_{c}$ is affected by the same sources of uncertainty as $P_{b}$, but the biggest contribution to its error is that due to the uncertainty in the amount of the hadron background in the lepton sample.

\subsection{Systematic Errors}

Basically three sources of systematic errors have to be considered for the mixed tag $R_{b}$ measurement:

a) uncertainties in the light quark efficiencies,

b) uncertainties in the correlation effects,

c) uncertainties in the knowledge of the composition of the lepton sample.

Errors from sources a) and b) were evaluated in exactly the same way as in section 4 . The effect of source a) turns out to be about a factor two smaller, since the light quark efficiencies enter only linearly in equations (8). The error on the correlation between the lepton tag and the vertex tag is dominated by the limited statistics available from the simulation. The two most important sources of correlation were gluon radiation and the correlated acceptances of the relevant detectors. For example, the hole between the barrel and forward muon chambers corresponds to a $\cos \theta$ region where the VD sensitivity is reduced; in the same way, the HPC polar acceptance overlaps with that of the VD (see section 2). In consequence, when a jet happened to fall near the border of the sensitive region of the VD, the probability of missing the lepton in the opposite hemisphere was higher. This induced a positive correlation between the two tags. 


\begin{tabular}{||l|l||}
\hline Source & $\Delta P_{b}$ \\
\hline Monte Carlo statistics & 0.31 \\
Lepton Fit & 0.36 \\
Model $b \rightarrow l$ & 0.39 \\
Model $c \rightarrow l$ & 0.31 \\
$b \rightarrow \tau \rightarrow l$ & 0.03 \\
$b \rightarrow \bar{c} \rightarrow l$ & 0.02 \\
$b \rightarrow J / \Psi \rightarrow l$ & 0.03 \\
$c \rightarrow l$ & 0.34 \\
$e$ misidentification & 0.12 \\
$\mu$ misidentification & 0.14 \\
$e$ identification efficiency & 0.02 \\
$\mu$ identification efficiency & 0.04 \\
\hline
\end{tabular}

Table 5: Systematic errors (\%) on the purity $P_{b}$ of the lepton sample when the selection $p_{t}^{\text {out }}>1.5 \mathrm{GeV} / \mathrm{c}$ was applied to the lepton transverse momentum.

The contributions due to the uncertainties in the purity of the lepton sample were then added to the total error. Table 6 gives the detailed contributions of all the sources of uncertainty considered above. Thus the final result from the 1993 analysis is

$$
R_{b}=0.2240 \pm 0.0039(\text { stat. }) \pm 0.0040(\text { syst. })-0.015 \frac{R_{c}-0.172}{0.172} .
$$

\begin{tabular}{||l|c||}
\hline Source of error & $\delta R_{b}$ \\
\hline Statistical & 0.0039 \\
$P_{b}$ & 0.0024 \\
$P_{c}$ & 0.0003 \\
Resolution Function & 0.0011 \\
Vertex-lepton correlations & 0.0022 \\
Charm efficiency & 0.0019 \\
$u d s$ efficiency & 0.0006 \\
\hline total & 0.0056 \\
\hline
\end{tabular}

Table 6: Contributions to the total error in the mixed tag analysis.

\subsection{Combination with the 1992 Analysis}

In order to combine the results of this analysis and the 1992 one [3], all the statistical uncertainties were treated as independent. For the combination of systematic errors:

- The errors on the two analyses due to gluon radiation were considered to be fully correlated, as were for those due to detector acceptance.

- The errors on the resolution functions were assumed to be independent, as for the lifetime analysis; the other uncertainties on the charm and light quarks efficiencies were treated as fully correlated. 
- The errors on the lepton purity due to limited Monte Carlo statistics were assumed to be independent. The uncertainties due to the heavy flavour decay models and branching ratios, and also the errors due to the lepton efficiencies and the background estimation, were treated as fully correlated.

With these assumptions, the result for the combined 1992/93 data is

$$
R_{b}=0.2233 \pm 0.0029(\text { stat. }) \pm 0.0035(\text { syst. })-0.015 \frac{R_{c}-0.172}{0.172} .
$$

\section{The Multivariate Analysis}

In the impact parameter analysis, hemispheres are tagged simply as $b$ and non- $b$. This leads to two equations with six unknowns, $R_{b}, \epsilon_{b}, R_{c}, \epsilon_{u d s}, \epsilon_{c}$ and $\rho_{b}$. Three of them, $\epsilon_{u d s}, \epsilon_{c}$ and $\rho_{b}$, are then taken from simulation and $R_{c}$ is fixed to the Standard Model value. If the number of equations for physical observables were larger than the number of unknowns, the latter could be extracted directly from the data, and the simulation would be required only to estimate systematic errors and the influence of hemisphere correlations. That is the principle of this method of measuring $R_{b}$, which uses the 1992 and 1993 data. The method was used previously to analyse the 1991 data [7]. The cut $\left|\cos \theta_{\text {thrust }}\right|<0.75$ was applied. This ensured that most of the tracks were within the acceptance of the microvertex detector. In order to reduce the correlation between opposite hemispheres as much as possible, a primary vertex was computed on each side; the position and dimensions of the beam spot were also used in these fits.

To provide the necessary hemisphere tagging categories (physical observables), an involved multivariate analysis technique is used. This tagging algorithm combines thirteen microvertex and event shape variables. The details of the technique and a full description of the variables can be found in [14] and were basically the same as those used for the 1991 analysis. One variable (boosted sphericity) is computed with four-momenta only, the remaining ones use the reconstructed trajectories near the interaction point. Three of them are connected to the $\chi^{2}$ fit of vertices associated with various sets of particles. Three are distances between "candidate secondary vertices" and the primary vertex, and are sensitive to decay lengths. Another three variables are different counters of "candidate secondary particles" and the last two are estimates of the total energy and $p_{t}^{2}$ associated with them. In order to improve the quality of the tagging as compared with that achieved in the 1991 analysis, the hemisphere probability variable $P_{H}$ used in the analyses presented in sections 4 and 5 was also included, but in this analysis it was constructed using the primary vertex computed separately in each hemisphere.

The probabilities $p_{u d s}, p_{c}$ and $p_{b}$ of observing the thirteen values of the variables for each hemisphere are computed from model distributions taken from simulation. These three probabilities are sorted in increasing order as $p_{\text {first }}, p_{\text {second }}$ and $p_{\text {third }}$. The hemispheres are first classified as $u d s, c$ or $b$ according to the highest probability, $p_{\text {first }}$. The $u d s$ tag is then subdivided into two categories (called uds-tight and uds-loose) and the $b$ tag into three categories ( $b$-loose, $b$-medium and $b$-tight). The categories are mutually exclusive and they have been ordered by increasing $b$ purity. The subdivision inside the $u d s$ and $b$ tags is based on a crucial classification criterion $\Delta$, called the winning margin, defined as

$$
\Delta=\ln \left(p_{\text {first }} / p_{\text {second }}\right),
$$


which is an indicator of tag clarity. The values of the cuts defining these categories were $\Delta_{u d s}^{c u t}=2.0, \Delta_{b}^{\text {cut }, \text { low }}=4.0$ and $\Delta_{b}^{\text {cut }, \text { high }}=8.0$. They were chosen in order to minimise the error.

The distribution of the winning margin $\Delta$ depends on the response of the tracking system, so that imperfect description of the detector accuracy could produce disagreement between data and simulation. The standard simulation described the distribution of the winning margin reasonably well, but not perfectly. The final effect on $R_{b}$ of the simulation not being fully realistic was expected to be small, because the efficiencies and backgrounds were estimated directly from the data, and almost independently of the simulation. Nevertheless, in order to improve this agreement, the different flavours in the simulated sample were weighted and moved around in such a way that the distribution in $\Delta$ for each tag coincided with that of the data sample. Even though this procedure does not ensure detailed agreement at the level of each flavour contribution separately, it improved the agreement in the description of the physical observables (defined in section 6.1) used to perform the $R_{b}$ measurement. Figure 8 shows the winning margin distribution in the $b$ tag after these corrections. Their final aim was only to improve the evaluation of the systematic errors.

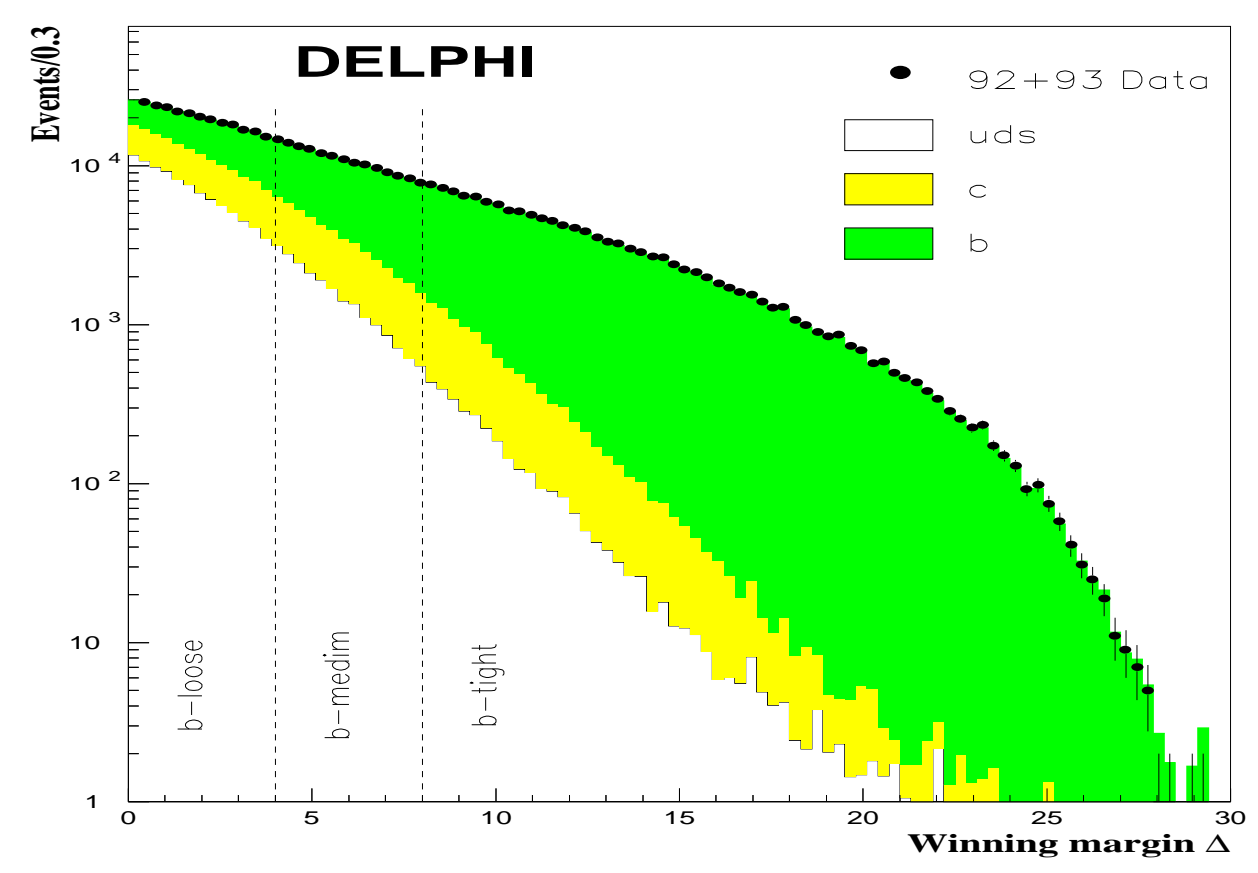

Figure 8: Distribution of the winning margin $\Delta$ in the $b$ tag categories for data and for simulation after correction. The different types of shading show the different flavour contributions to the simulated event sample. The simulation distributions are normalized to the data statistics. The values of the cuts defining the three $b$ tag categories are also indicated.

\subsection{The fit procedure}

The mathematical formalism of the fit procedure is described in reference [14]. The tagging algorithm classified the $N_{F}=3$ flavours ( $u d s, c$ and $b$ ) of the hadronic events into 
$N_{T}$ categories. Even though the smallest number of categories to measure $R_{b}$ with this method is $N_{T}=4$, the choice $N_{T}=6$ was made in order to overconstrain the problem and to minimise the error.

The first set of observables was the matrix $D_{I J}\left(\mathrm{I}, \mathrm{J}=1, \ldots, N_{T}\right)$, defined as the observed fraction of events tagged as $\mathrm{I}$ and $\mathrm{J}$ for hemispheres 1 and 2 respectively. The corresponding expected fraction of events $T_{I J}$ can be written as

$$
T_{I J}=\sum_{q} \epsilon_{I}^{q} \epsilon_{J}^{q}\left(1+\rho_{J I}^{q}\right) R_{q} .
$$

In equation (12), the $R_{q}$ are the flavour fractions and $\epsilon_{I}^{q}$ is the probability to classify a hemisphere of flavour $q$ in category I. The $6 \times 3$ array $\epsilon_{I}^{q}$, called the classification matrix, was assumed to be the same for both hemispheres. In a first approximation, the probability to classify an event of a flavour $q$ in one hemisphere is independent of the classification in the other hemisphere. In order to take inter-hemisphere correlations into account, the matrix $\rho_{J I}^{q}$ was introduced. The values of these elements for $b$ quarks, as estimated from simulation, are shown in figure $9(\mathrm{a})$. Most of them are small, or else are not significant for the extraction of $R_{b}$.
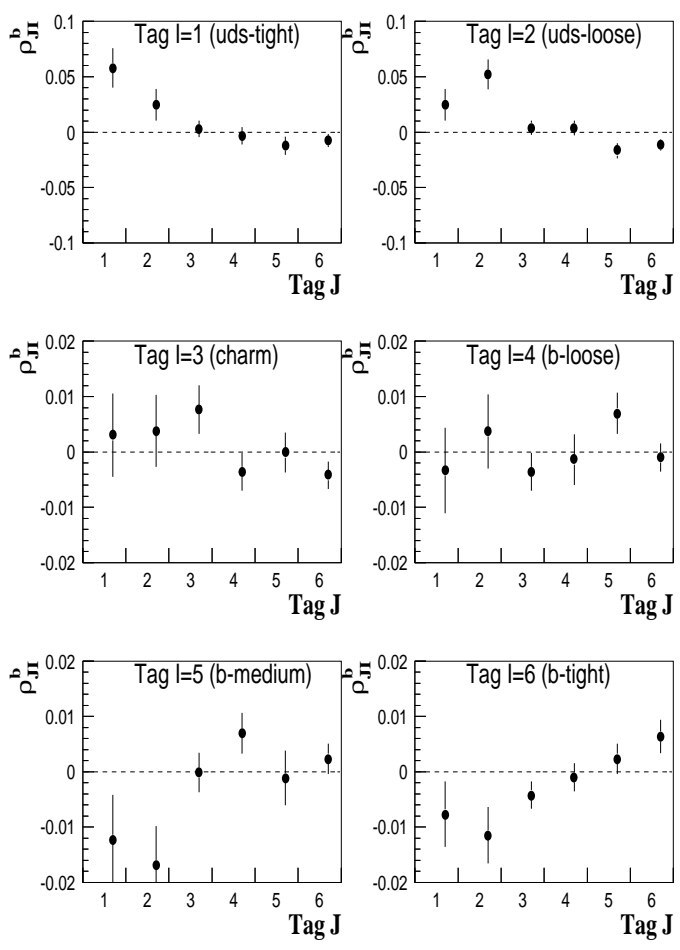

(a) Matrix correlation factors $\rho_{J I}^{b}$.

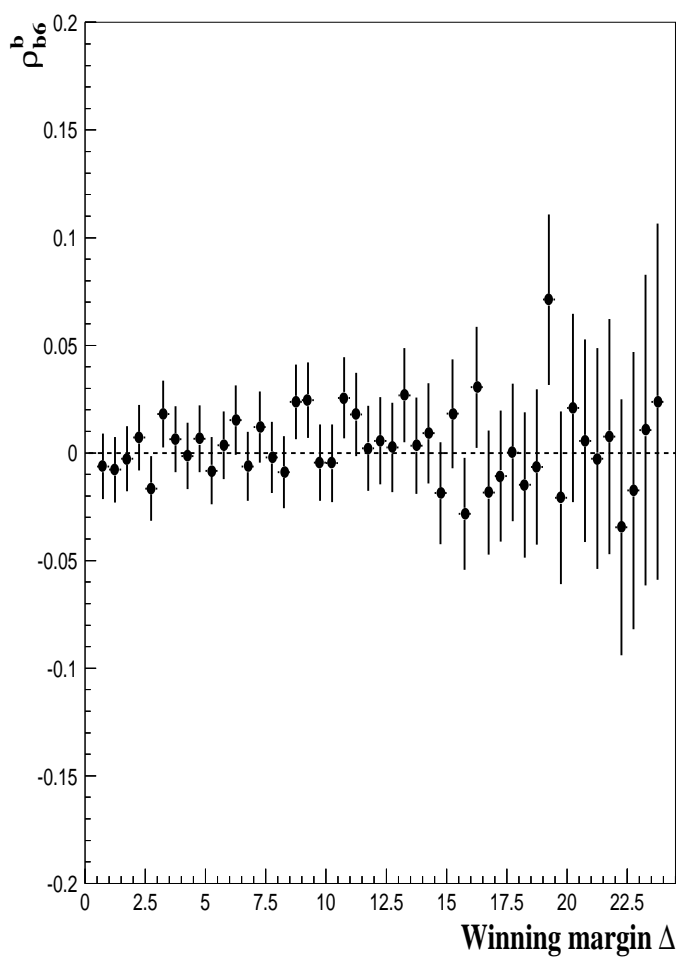

(b) Running correlation factor $\rho_{b 6}^{b}(\Delta)$.

Figure 9: a) Double tag hemisphere correlation factors for $b$ quarks estimated from simulation, b) dependence on the winning margin $\Delta$ of the most important correlation factor, $\rho_{b 6}^{b}(\Delta)$.

It is not possible to extract $R_{b}$ by a simple fit of the expected fractions $T_{I, J}$ to the observed fractions $D_{I J}$ because of the rotation degeneracy described in [14]. To solve this 
problem, a second set of observables, the distributions of the category fractions $f_{I}(\Delta)$, was used. $f_{I}(\Delta)$ is defined as the fraction of the events tagged as $b$ in one hemisphere with a winning margin in a given bin of $\Delta, \Delta \pm \delta$, that are classified in category $\mathrm{I}$ in the other hemisphere. The main property of the fraction $f_{I}(\Delta)$ is that its asymptotic value provides an estimate of the corresponding classification probability $\epsilon_{I}^{b}$.

The correlations in the background terms were found to have only a small influence, and were neglected. Then the $f_{I}(\Delta)$ fractions can be expressed as

$$
f_{I}(\Delta)=\epsilon_{I}^{b}\left\{1+\rho_{b I}^{b}(\Delta)\right\}+\xi_{I}(\Delta)
$$

with

$$
\xi_{I}(\Delta)=\left(\epsilon_{I}^{u d s}-\epsilon_{I}^{b}\right) \mathcal{R}_{u d s}(\Delta)+\left(\epsilon_{I}^{c}-\epsilon_{I}^{b}\right) \mathcal{R}_{c}(\Delta) .
$$

where $\mathcal{R}_{u d s}(\Delta)$ and $\mathcal{R}_{c}(\Delta)$ are the $u d s$ and $c$ contaminations in the $b$-tagged hemisphere and are independent of the index I. From (13) and (14), the asymptotic value of $f_{I}(\Delta)$ is $\left\{1+\lim _{\Delta \rightarrow \infty} \rho_{b I}^{b}(\Delta)\right\} \epsilon_{I}^{b}$, provided high purity is achieved in the $b$-tagged hemisphere for large values of $\Delta$. That this high purity is indeed achieved can be seen in figure 8 . The coefficients $\rho_{b I}^{b}(\Delta)$ in 13 are the running hemisphere correlation coefficients as a function of $\Delta$ for each tag I. The main correlation factor for the $R_{b}$ measurement is $\rho_{b 6}^{b}$. Figure 9 (b) shows the variation of this coefficient with $\Delta$ in the simulated data. Within the statistical errors it is very stable, even at large values of $\Delta$, and is always compatible with zero.

In order to extract the asymptotic value of $f_{I}(\Delta)$, an analytical parametrization of the $\xi_{I}(\Delta)$ distributions must be used. It was found that the parametrization which best describes the whole range of the contamination distributions $\mathcal{R}_{u d s}(\Delta)$ and $\mathcal{R}_{c}(\Delta)$ for the DELPHI data is the product of an exponential with a Gaussian function.

In the 1991 analysis, equation (13) was used to fit the fractions $f_{I}(\Delta)$ to extract their asymptotes, $\epsilon_{I}^{b}$. The values of $\epsilon_{I}^{b}$ were then inserted into a final fit to the matrix $D_{I J}$ to extract $R_{b}$ [7]. The problem with this technique is to evaluate properly systematic errors in the estimates of $\epsilon_{I}^{b}$. This difficulty has been avoided in the present analysis by combining the two fits into one by minimizing the global objective $\chi^{2}(\epsilon, R)$ function, defined as

$$
\chi^{2}(\epsilon, R)=\sum_{I J} \frac{\left\{D_{I J}-T_{I J}\right\}^{2}}{\sigma_{I J}^{2}}+\sum_{I, \Delta} \frac{\left\{f_{I}(\Delta)-\epsilon_{I}^{b}\left[1+\rho_{b I}^{b}(\Delta)\right]-\xi_{I}(\Delta)\right\}^{2}}{\sigma_{f_{I}(\Delta)}^{2}} .
$$

This allows the simultaneous determination of the classification matrix, the contamination distributions $\xi_{I}(\Delta)$ and $R_{b}$. The $\sigma_{I J}$ are the statistical errors of the $D_{I J}$ elements and $\sigma_{f_{I}(\Delta)}$ are the experimental errors on $f_{I}(\Delta)$ for each bin of $\Delta$. With this function and in the absence of correlations, a degeneracy in the $u d s c$ sector is still present but it can be removed, for instance, by fixing $R_{c}$ to the Standard Model value. This constraint has no effect on any parameter of the $b$ sector [14].

Another advantage of this global fit is that it provides the unique solution that reproduces the matrix population $D_{I J}$ and the $f_{I}(\Delta)$ fractions simultaneously. In addition, hemisphere correlation effects can be studied simultaneously for both terms of equation (15). The disadvantage is the introduction of a relatively large number of auxiliary parameters in the minimization procedure. 


\section{2 $R_{b}$ measurement and consistency checks}

The data samples collected in 1992 and 1993 were analyzed independently because differences in the microvertex detector were expected to result in slightly different tagging efficiencies. About 1,400,000 hadronic $Z$ decays were selected, of which 974,149 passed the $\left|\cos \theta_{\text {thrust }}\right|$ cut. The bias of the $Z \rightarrow b \bar{b}$ fraction in the selected sample was estimated from simulation and was found to be small, $0.0005 \pm 0.0004$ for the 1992 data and $0.0009 \pm 0.0004$ for 1993 . The plots of the $f_{I}(\Delta)$ distributions as a function of the winning margin $\Delta$ are shown in figure 10 for the combined 1992 and 1993 data. The reproducibility and reliability of the method was tested by analyzing the same simulated events that were used to estimate the correlation coefficients. Their $f_{I}(\Delta)$ distributions are also shown in figure 10, together with the separate contributions of $u d s, c$ and $b$ flavours.

Categories 1 (uds-tight) and 2 (uds-loose) contain the smallest fractions of $b$ hemispheres, as can be seen from the higher $u d s$ and $c$ backgrounds in the distributions of $f_{1}(\Delta)$ and $f_{2}(\Delta)$; to achieve high $b$ purity thus requires tighter cuts in $\Delta$ than in the other categories. However these categories have rather little weight in the evaluation of $R_{b}$, since they account for only about $4 \%$ and $7 \%$ respectively of the $b$ hemispheres in the data.

No significant irreducible $u d s$ and $c$ background is observed in the asymptotic regions of the $f_{4}(\Delta), f_{5}(\Delta)$ and $f_{6}(\Delta)$ distributions, which are the most significant for the $R_{b}$ extraction. Effects of the remaining background are small and are included in the systematic uncertainties.

In the fit of the $\chi^{2}(\epsilon, R)$ function, the $R_{c}$ parameter was fixed to the Standard Model value. Even though there were many free parameters in the fit, no subsidiary local minima were seen in the whole range of $R_{b}$. Table 7 summarizes the $\epsilon_{I}^{b}$ and $R_{b}$ values fitted to simulated and real data taking hemisphere correlations into account, and also the values expected for the simulated data. The $\epsilon_{u d s-t i g h t}^{b}$ element is the least well reproduced. Background effects were not negligible in this $b$-depleted category and $2.5 \sigma$ differences were observed. However this matrix element was not significant in the $R_{b}$ extraction. Good agreement was found between the expected and fitted values for all the other $\epsilon_{I}^{b}$ parameters, and for the $\epsilon_{I}^{u d s}$ and $\epsilon_{I}^{c}$.

Table 7 shows that the difference between the generated and the fitted $R_{b}$ is $0.0018 \pm$ 0.0027 in the 1992 simulation and $-0.0007 \pm 0.0026$ in the 1993 one. On average, the measured values agree within $0.0005 \pm 0.0018$ with the expected ones, so it may be concluded that the method produces no bias on the measurement.

\begin{tabular}{|c|c|c|c|c|c|c|c|}
\hline \multirow[b]{2}{*}{ Categ } & \multirow[b]{2}{*}{ Param. } & \multicolumn{2}{|c|}{1992 Simulation } & \multirow{2}{*}{$\begin{array}{c}1992 \text { Data } \\
\text { Fitted }\end{array}$} & \multicolumn{2}{|c|}{1993 Simulation } & \multirow{2}{*}{$\begin{array}{c}1993 \text { Data } \\
\text { Fitted }\end{array}$} \\
\hline & & Expected & Fitted & & Expected & Fitted & \\
\hline 1 & & 0.0501 & $0.0529(11)$ & $0.0562(20)$ & 0.0454 & $0.0470(10)$ & $0.0354(18)$ \\
\hline 2 & & 0.0801 & $0.0818(10)$ & $0.0802(15)$ & 0.0823 & $0.0830(10)$ & $0.0592(21)$ \\
\hline 3 & & 0.2030 & $0.2023(19)$ & $0.2046(26)$ & 0.2005 & $0.1980(19)$ & $0.1824(24)$ \\
\hline 4 & $\epsilon_{b-l c}^{b}$ & 0.2207 & $0.2195(10)$ & $0.2074(18)$ & 0.2136 & $0.2136(08)$ & $0.2140(17)$ \\
\hline 5 & $\epsilon_{b-m e}^{b}$ & 0.1924 & $0.1908(11)$ & $0.1864(16)$ & 0.1969 & $0.1966(09)$ & $0.2006(15)$ \\
\hline 6 & $\epsilon_{b-t}^{b}$ & 0.2537 & $0.2523(17)$ & $0.2653(30)$ & 0.2613 & $0.2619(14)$ & $0.3084(34)$ \\
\hline & $R_{b}$ & 0.217 & $0.2188(27)$ & $0.2163(51)$ & 0.217 & $0.2163(26)$ & $0.2209(51)$ \\
\hline & $\operatorname{Prob}\left(\chi^{2}\right)$ & & $9.5 \%$ & $23.1 \%$ & & $33.3 \%$ & $10.9 \%$ \\
\hline
\end{tabular}

Table 7: Results of the 1992/1993 simulation and real data fits using the correlation pattern taken from the simulation. For comparison, the values expected for the simulations are also shown. The statistical errors are given in brackets and affect the last two digits. 


\section{DELPHI}
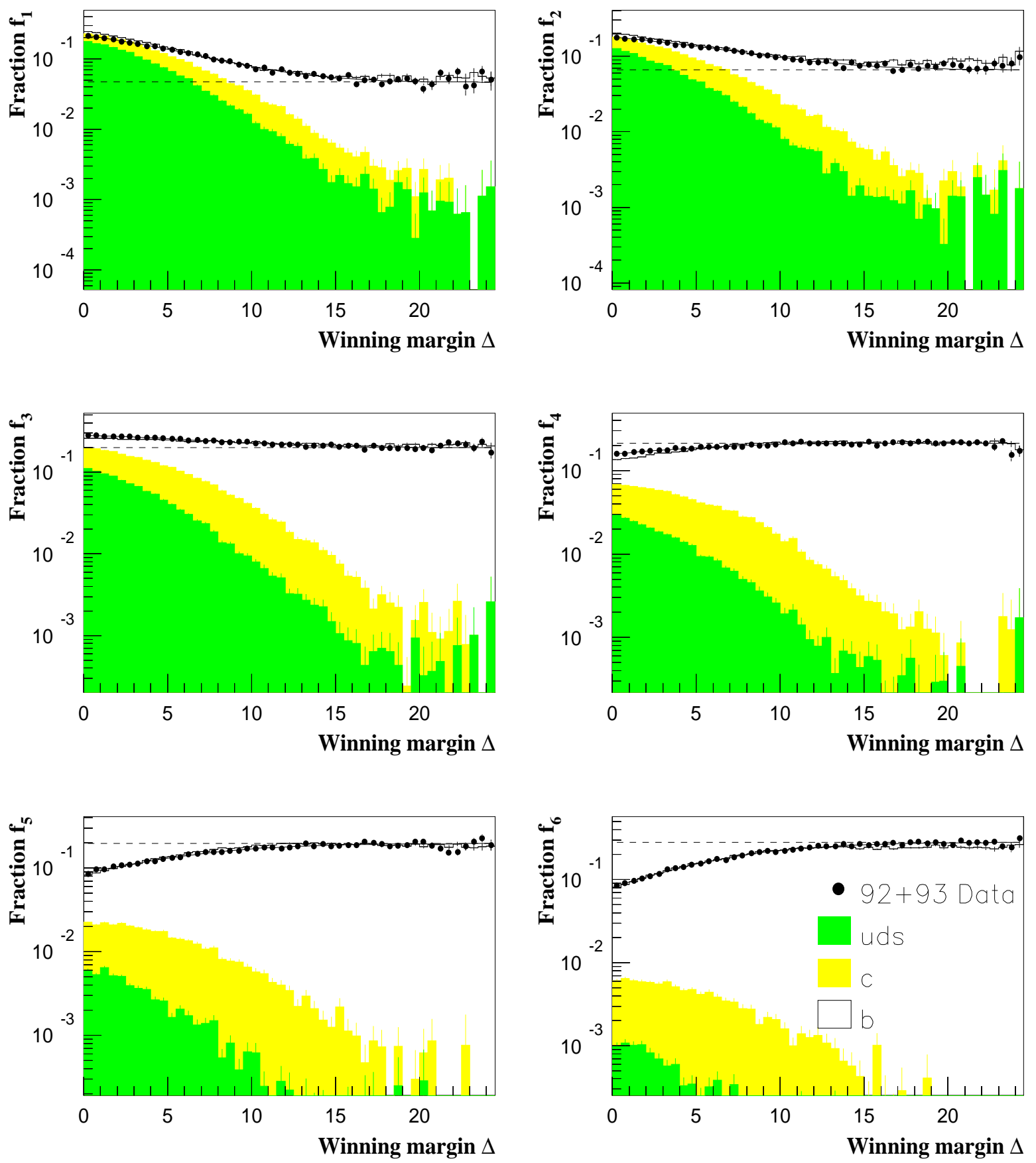

Figure 10: The distributions of the fractions $f_{I}(\Delta)$ with their fits for the combined $92+93$ data. The dashed horizontal lines show the $\epsilon_{I}^{b}$ values fitted from the data. The distributions for the simulation are also shown, together with the contributions of $u d s$, $c$ and $b$ flavours. To show the small backgrounds in the region of hard cuts, the vertical scale on each plot goes down to one per mil of the fitted value of $\epsilon_{I}^{b}$. 
The off peak and on peak data are expected to have almost the same fraction of $b \bar{b}$ events, since the hadronic cross sections at these energies are still dominated by $Z$ exchange. Analyses of the off peak and on peak data taken in 1993 gave $R_{b}(89.49 \mathrm{GeV})=$ $0.2220 \pm 0.0157, R_{b}(91.25 \mathrm{GeV})=0.2210 \pm 0.0059$ and $R_{b}(93.08 \mathrm{GeV})=0.2186 \pm$ 0.0139. The corresponding $\chi^{2}$ probabilities of the fits were $45.3 \%, 74.5 \%$ and $37.6 \%$ respectively. As the differences between the on peak and off peak values of $R_{b}$ are not sensitive to systematic effects, these numbers can be combined using only statistical errors. Before averaging them, the off peak values of $R_{b}$ were corrected for the small differences expected relative to the on peak value. These corrections were predicted by ZFITTER [15] and changed the off peak values by 0.0007 and 0.0005 for $\sqrt{s}=89.49 \mathrm{GeV}$ and $\sqrt{s}=93.08 \mathrm{GeV}$ respectively. The value for 1993 data in table 7 is the result of combining the three energies after these corrections. It agrees within three per mil with the result obtained when all the statistics were analyzed together, which is a consistency check of the reproducibility and reliability of the method.

As a cross-check on the effect of correlations, the fits shown in table 7 were repeated taking all correlation coefficients equal to zero. The smallness of the change in the results was remarkable: the change in $R_{b}$ was only $0.00103 \pm 0.00161$ for the 1992 data and $-0.00146 \pm 0.00150$ for the 1993 data, where the errors are computed from the change in $R_{b}$ obtained by moving the correlation coefficients by their statistical errors. This demonstrates the insensitivity of the method to the predicted pattern of correlations.

As another cross-check on the stability of $R_{b}$, the measurement was repeated for different tagging cuts defining the three $b$ categories. Figure 11 shows the difference of each measurement with respect to the reference result as a function of $\Delta_{b}^{\text {cut } \text {, high }}$, taking $\Delta_{b}^{\text {cut }, \text { low }}$ to be always half of $\Delta_{b}^{c u t, h i g h}$. The measured value is stable over a wide range of variation of the efficiencies inside each $b$ category. Moreover, $R_{b}$ was also measured using the minimal configuration of $N_{T}=4$ categories and the difference observed with respect to the reference result was 0.0011 , well inside the statistical error on the difference of 0.0029 .

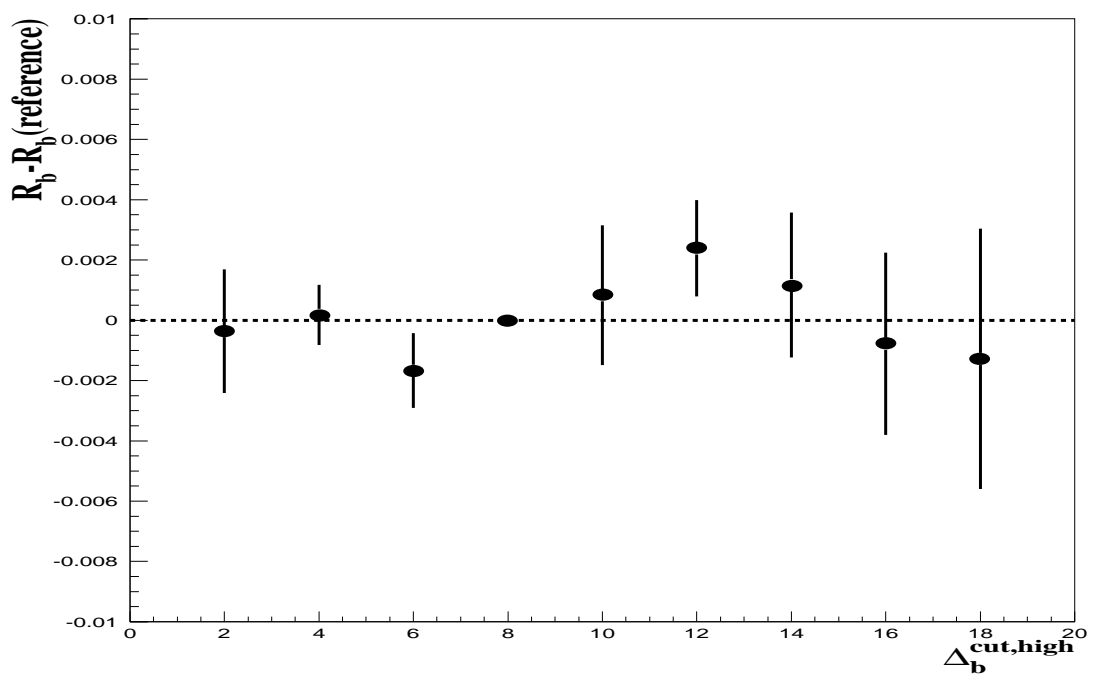

Figure 11: The value of $R_{b}-R_{b}$ (reference), with its error, as a function of the cuts defining the three b categories. $\Delta_{b}^{\text {cut }, \text { low }}$ was taken as half of $\Delta_{b}^{\text {cut }, \text { high }}$. The reference value of $R_{b}$ is the value quoted in the text. 


\subsection{Systematic errors}

Three kinds of systematic errors were studied separately: uncertainties coming from models, from detector effects and from the analysis method.

\subsubsection{Model uncertainties}

Most methods of $R_{b}$ determination assume the knowledge of the $b$-tagging backgrounds $[1-5]$. These quantities, taken from simulation, are sensitive to theoretical uncertainties in the $u d s$ and $c$ sectors and are sources of systematic error. In this method, $R_{b}$ is extracted simultaneously with the efficiencies and backgrounds by fitting the data. However, the analysis assumes that for asymptotically hard cuts the contributions of $u d s$ and $c$ are negligible. In the absence of hemisphere correlations and remaining $u d s$ and $c$ background in the region of hard cuts, the $R_{b}$ measurement is mathematically independent of the factors that affect $b$ production or decay, for example fragmentation functions or lifetimes. In that case the corresponding systematic errors are exactly zero. If the hypothesis is almost true, second order effects on the $R_{b}$ measurement can appear and should be included in the systematic uncertainties.

In the previous section it was shown that only a small difference in $R_{b}$ is observed if the estimated correlation matrix is taken into account in the fit or if it is neglected. This suggests that the method is insensitive to the particular pattern of correlations. There is no evidence for a fundamentally different correlation pattern in real data compared to the simulation. The error made on data coming from correlations should be similar to the one made on the simulation. An estimate of this error was obtained by varying the parameters of the simulation that could be sources of correlation.

By following the prescriptions described in reference [6], we have checked that errors due to modelling are of second order. Table 9 summarizes all the contributions to the systematic error coming from model uncertainties.

Correlation effects can be described in terms of the following sources:

- Hadronic $Z$ events with three or more jets differ from those with a two jet topology by the presence of one or more hard gluons in the final state. This effect includes the hard gluon emission producing a $b \bar{b}$ pair in the same hemisphere. To estimate the systematic error from this source, the number of events was measured in data and in simulation as a function of the thrust of the event. The simulation thrust distribution was then corrected to reproduce the data distribution. The error was estimated as the change in the fitted value of $R_{b}$, due to the change of correlations and efficiencies, between the standard simulation and the corrected one. The magnitude of the effect was 0.00061 .

- The bias of the production vertex due to the inclusion of tracks from $b$ decays can produce a negative correlation. The lifetime of $b$-hadrons and the $b$ fragmentation function are the most relevant parameters. The change in the correlations resulting from a change of the $b$ lifetime was estimated by applying different decay time weighting functions to the simulated event sample. The change in the correlations led to a change in $R_{b}$ of 0.00022 . The uncertainty due to the $b$ fragmentation function was estimated similarly, by varying the Peterson parameter to reproduce the mean energy of B-hadrons within its error limits. The resultant error was 0.00038 .

- Finally, the uncertainties in the correlations coming from the limited simulation statistics were included. 
For the evaluation of the systematic uncertainty coming from the remaining uds and $c$ background, the following contributions were considered:

- The dependence of $R_{b}$ on the fraction of charm events (which should be distinguished from the formal $R_{c}$ parameter of the fit) was estimated by changing the $c \bar{c}$ fraction in the simulation around its Standard Model value. A dependence of $-0.0049 \frac{R_{c}-0.172}{0.172}$ was found. In contrast, when the $R_{c}$ fit parameter was varied the change of $R_{b}$ was exactly zero.

- The uncertainty due to the $c$ fragmentation function was estimated similarly, by varying the Peterson parameter to reproduce the uncertainty in the mean energy of D-hadrons.

- The uncertainties from the relative production rates of D-hadrons, their lifetimes, their decay multiplicities and their inclusive branching ratios $D \rightarrow K^{0} X$ were obtained by varying these values about the measured ones according to [6].

- The systematic error from uncertainties in the production of long-lived particles in light quark events ( $K^{0}, \Lambda$, hyperons) was obtained by varying the corresponding production rates in the simulation by $\pm 10 \%$.

- The systematic errors from uncertainties on the gluon splitting processes $g \rightarrow b \bar{b}$ and $g \rightarrow c \bar{c}$ were obtained by varying the fraction of such events by $50 \%$.

To obtain the systematic error from these sources, the Monte Carlo simulated events were weighted as a function of the relevant model parameter. The weighted simulated sample was then fitted, and the difference with respect to the fit to the standard simulated sample was taken as the error.

\subsubsection{Detector effects}

The detector effects include all sources of uncertainties due to the apparatus and can be described in the following terms:

- Detector response. Differences between data and simulation are not important in the present analysis, because all efficiencies and backgrounds are obtained directly from the data; only a small model dependence remains due to hemisphere correlation effects and the possible background remaining in the region of hard cuts. As explained in section 6, the Monte Carlo sample was corrected to adjust the winning margin distribution to the data. This procedure improves the agreement between data and simulation at the level of the $D_{I J}$ matrix and the $f_{I}(\Delta)$ distributions. For the uncertainty due to the knowledge of the detector response we therefore take the difference between the measurements using the standard simulated sample and the corrected one, and add in quadrature the error on this difference. The values obtained were 0.00038 for the 1992 sample and 0.00089 for 1993 .

- The polar angle of the thrust axis. Correlation effects could be induced by the fall in tag efficiency at the fringes of the vertex detector acceptance, where both jets are in a region of less good VD acceptance simultaneously. To obtain the systematic error from this source, the number of $b$-tagged events was measured in data and in simulation as a function of $\left|\cos \theta_{\text {thrust }}\right|$. The simulation distribution was corrected in order to reproduce the corresponding data distribution and the effect on $R_{b}$ was determined. A contribution of 0.00042 for 1992 and 0.00041 for 1993 is quoted, using the same method as for the detector response.

- The azimuthal angle of the jets. Due to dead or noisy modules in the vertex detector, the efficiency was not independent of the azimuthal angle. In particular, during 
the 1992 running, one row of the DELPHI vertex detector in one layer was dead. In an almost back to back jet topology, hitting a bad module on one side then normally results in hitting a good module on the other side, producing a negative correlation. The multivariate tagging is not sensitive to local defects, so the variation of the tag efficiency with the azimuthal direction of the event axis is not important. Nevertheless, we have investigated the error due to the local drop of efficiency, which induces a small negative correlation. The method used was the same as for the polar angle correlation. Contributions of 0.00034 for 1992 data and 0.00009 for 1993 data were found.

- Beam spot constraint. This constraint can be a source of correlations owing to the beam spot size, since the beam spot constraint is common to both hemispheres. A $10 \%$ change (which corresponds to the accuracy of the size determination) was found to change $R_{b}$ by 0.00034 for both 1992 and 1993 data.

\subsubsection{Analysis method}

The fit to the simulation discussed in section 6.2 showed that the analysis method is unbiassed, within the accuracy of the limited Monte Carlo statistics. In particular, it was shown that the difference between the generated and the fitted $R_{b}$ is $0.0018 \pm 0.0027$ in 1992 , and $-0.0007 \pm 0.0026$ in 1993 . On the other hand, as was indicated in section 6.1 , the method assumes that estimates of the $\epsilon_{I}^{b}$ column of the classification matrix can be extracted asymptotically. The effect of this assumption can be tested by fitting $R_{b}$ in

the simulation with the $\epsilon_{I}^{b}$ parameters fixed to their true values. The difference obtained with respect to the full measurement was $0.0011 \pm 0.0022$ for 1992 and $-0.0001 \pm 0.0021$ for 1993, where the errors are due to Monte Carlo statistics. These values were used to correct the $R_{b}$ derived from the fits to the data, and their errors were taken as a systematic uncertainty on the measurement due to the analysis method. This becomes the most important contribution to the systematic error which is uncorrelated between the different years; it could be reduced with more simulation statistics.

Therefore we quote as final values, including acceptance and systematic corrections,

$$
R_{b}=0.2152 \pm 0.0051(\text { stat. }) \pm 0.0030 \text { (syst.) }
$$

and

$$
R_{b}=0.2210 \pm 0.0051 \text { (stat.) } \pm 0.0030 \text { (syst.) }
$$

for 1992 and 1993 data respectively.

\subsection{Combination of the 1991 to 1993 results}

In order to combine the analyses presented here with the corresponding one made with the 1991 data, the following assumptions are made.

- All statistical errors are assumed to be independent, including the data statistical error and the simulation statistical errors on the checks of the effects of correlations and the analysis method.

- The errors due to model uncertainties on efficiency correlations and $b$ tag backgrounds are taken to be fully correlated.

- The error from acceptance bias was assumed to be uncorrelated.

- All other errors from detector effects were taken to be zero in the 1991 analysis, because they were assumed to be well described within the statistical error from the 


\begin{tabular}{||l|c|c|c|c||}
\hline Error Source & \multicolumn{4}{|c||}{ Uncertainty $\times 10^{4}$} \\
& 91 data & 92 data & 93 data & combined \\
\hline Statistical error & \pm 63.0 & \pm 50.9 & \pm 51.0 & \pm 31.8 \\
\hline Model uncertainties & & & & \pm 8.4 \\
Simulation statistics on correlations & \pm 27.7 & \pm 16.1 & \pm 15.0 & \pm 10.4 \\
Detector response & \pm 8.7 & \pm 3.8 & \pm 8.9 & \pm 6.8 \\
Polar angle acceptance & \pm 7.5 & \pm 4.2 & \pm 4.1 & \pm 4.8 \\
Azimuthal angle acceptance & \pm 9.3 & \pm 3.4 & \pm 0.9 & \pm 3.6 \\
Beam spot size & & & & \pm 3.4 \\
Acceptance bias & \pm 7.0 & \pm 4.3 & \pm 3.7 & \pm 2.7 \\
Analysis method & \pm 38.4 & \pm 22.2 & \pm 21.0 & \pm 14.5 \\
\hline Total systematic error & \pm 51.0 & \pm 30.1 & \pm 29.6 & \pm 22.2 \\
\hline Total error & \pm 81.0 & \pm 59.1 & \pm 59.0 & \pm 38.9 \\
\hline
\end{tabular}

Table 8: Breakdown of the error on $R_{b}$ obtained from the multivariate tagging for each year, and on the combined value. Common systematic errors are only given in the column of the combined analysis.

fit to the simulation. In order to be consistent in the average, these errors have been recomputed using the method described above. Finally, they were conservatively assumed to be fully correlated.

With these assumptions the final result is

$$
R_{b}=0.2194 \pm 0.0032(\text { stat. }) \pm 0.0022(\text { syst. })-0.0049 \frac{R_{c}-0.172}{0.172} .
$$

The breakdown of the errors on the separate and the combined results are given in table 8.

\section{Combination of the Results}

The results from the different analyses have been combined taking into account the common systematic errors. The breakdown of the errors for the individual analyses and for the combination is given in table 9 . The errors within a line have been assumed to be fully correlated. The statistical correlation between the mixed tag and the other analyses can be neglected. The correlation between the double impact parameter tag and the multivariate analysis has been estimated using a Monte Carlo technique to be less than 0.35 (90\% C.L.). Conservatively this value has been used in the average ${ }^{\dagger}$. The combined result is:

$$
R_{b}=0.2213 \pm 0.0016(\text { stat. }) \pm 0.0021(\text { syst. })-0.015 \frac{R_{c}-0.172}{0.172}
$$

with $\chi^{2} / n d f=0.5 / 2$.

Because of the different charges of up-type and down-type quarks, a correction of +0.0003 due to photon exchange has to be applied to obtain $R_{b}^{0}$ from $R_{b}$ [15], resulting in

$$
\left.R_{b}^{0}=0.2216 \pm 0.0016(\text { stat. }) \pm 0.0021 \text { (syst. }\right)-0.015 \frac{R_{c}-0.172}{0.172} .
$$

\footnotetext{
${ }^{\dagger}$ The most probable value for the correlation was found to be 0 . It has been checked that the final result does not change using this value.
} 


\begin{tabular}{|c|c|c|c|c|c|}
\hline \multirow[b]{2}{*}{ Error Source } & \multirow[b]{2}{*}{ Range } & \multicolumn{4}{|c|}{ Uncertainty $\times 10^{4}$} \\
\hline & & $\operatorname{dit}$ & $\mathrm{mt}$ & mult & com. \\
\hline Internal experimental effects: & & & & & \\
\hline Hemisphere correlations & & \pm 11 & 0 & \pm 14.4 & \pm 8 \\
\hline Lepton-vertex correlations & & 0 & \pm 13 & 0 & \pm 3 \\
\hline Resolution function & & \pm 9 & \pm 9 & \pm 6.8 & \pm 8 \\
\hline Lepton sample purity & & 0 & \pm 19 & 0 & \pm 4 \\
\hline Acceptance bias & & \pm 2 & 0 & \pm 2.7 & \pm 1 \\
\hline Method & & 0 & 0 & \pm 14.5 & \pm 5 \\
\hline$\left\langle x_{E}(c)\right\rangle$ & $0.49 \pm 0.02$ & $\mp 5$ & $\mp 5$ & \pm 1.5 & $\mp 4$ \\
\hline $\mathrm{Br}(c \rightarrow \ell)$ & $(9.8 \pm 0.5) \%$ & 0 & \pm 10 & 0 & \pm 2 \\
\hline Semilept. model $b \rightarrow \ell[6]$ & $\left(\begin{array}{l}+\mathrm{ACCMM} \\
-\mathrm{ISGW} * *\end{array}\right)$ & 0 & \pm 11 & 0 & \pm 2 \\
\hline Semilept. model $c \rightarrow \ell[6]$ & ACCMM1 $\left({ }_{-A C C M M}^{+\mathrm{ACCMM}}\right)$ & 0 & $\mp 8$ & 0 & $\mp 2$ \\
\hline$D^{0}$ fraction in $c \bar{c}$ events & $0.557 \pm 0.053$ & $\mp 1$ & $\mp 1$ & $\mp 0.2$ & $\mp 1$ \\
\hline$D^{+}$fraction in $c \bar{c}$ events & $0.248 \pm 0.037$ & $\mp 15$ & $\mp 8$ & $\mp 0.5$ & $\mp 9$ \\
\hline$\left(D^{0}+D^{+}\right)$fraction in $c \bar{c}$ events & $0.80 \pm 0.07$ & $\mp 8$ & $\mp 5$ & $\mp 0.6$ & $\mp 5$ \\
\hline$D_{s}$ fraction in $c \bar{c}$ events & $0.12 \pm 0.05$ & $\mp 7$ & $\mp 7$ & $\mp 3.4$ & $\mp 6$ \\
\hline$D^{0}$ lifetime & $0.420 \pm 0.008 \mathrm{ps}$ & $\mp 3$ & $\mp 2$ & $\mp 0.4$ & $\mp 2$ \\
\hline$D^{+}$lifetime & $1.066 \pm 0.023 \mathrm{ps}$ & $\mp 4$ & $\mp 2$ & $\mp 0.3$ & $\mp 2$ \\
\hline$D_{s}$ lifetime & $0.450_{-0.026}^{+0.030} \mathrm{ps}$ & $\mp 3$ & $\mp 2$ & $\mp 0.4$ & $\mp 2$ \\
\hline$\Lambda_{c}$ lifetime & $0.191_{-0.012}^{+0.015} \mathrm{ps}$ & 0 & 0 & $\mp 0.7$ & 0 \\
\hline D decay multiplicity & $2.53 \pm 0.06$ & $\mp 6$ & $\mp 4$ & $\mp 0.2$ & $\mp 4$ \\
\hline$B R\left(D \rightarrow K^{0} X\right)$ & $0.46 \pm 0.06$ & \pm 8 & \pm 7 & \pm 0.5 & \pm 6 \\
\hline $\mathrm{g} \rightarrow b \bar{b}$ per multihadron & $(0.16 \pm 0.08) \%$ & $\mp 3$ & $\mp 3$ & $\mp 0.1$ & $\mp 2$ \\
\hline$g \rightarrow c \bar{c}$ per multihadron & $(1.5 \pm 0.8) \%$ & $\mp 1$ & $\mp 1$ & $\mp 0.1$ & $\mp 1$ \\
\hline Rate of long-lived light hadrons & Tuned JETSET $\pm 10 \%$ & $\mp 6$ & $\mp 5$ & $\mp 0.4$ & $\mp 4$ \\
\hline
\end{tabular}

Table 9: Summary of systematic errors on $R_{b}$ obtained from the double impact parameter tag (dit, section 4), the mixed tag (mt, section 5), the multivariate tag (mult, section 6) and the combination of the three analyses. Detailed explanations how the different error sources are obtained can be found in [6].

\section{Conclusions}

Three different measurements of the partial decay width $R_{b}^{0}$ of the $Z$ into B-hadrons have been performed. Events were selected using either tracks having large impact parameters or leptons with high transverse momentum. The following results were obtained: Double impact parameter tag:

$$
R_{b}=0.2219 \pm 0.0018(\text { stat. }) \pm 0.0028(\text { syst. })-0.022 \frac{R_{c}-0.172}{0.172}
$$

Mixed tag:

$$
R_{b}=0.2233 \pm 0.0029(\text { stat. }) \pm 0.0035(\text { syst. })-0.015 \frac{R_{c}-0.172}{0.172}
$$

Multivariate analysis:

$$
R_{b}=0.2194 \pm 0.0032(\text { stat. }) \pm 0.0022(\text { syst. })-0.0049 \frac{R_{c}-0.172}{0.172}
$$


Combining all numbers and correcting for photon exchange gave

$$
\left.R_{b}^{0}=0.2216 \pm 0.0016(\text { stat. }) \pm 0.0021 \text { (syst. }\right)-0.015 \frac{R_{c}-0.172}{0.172}
$$

For this number, all centre of mass energies at which LEP has run have been combined. All results are in agreement with those of other measurements at LEP $[1,2,4,5]$. Assuming a mass of the top quark of $m_{t}=180 \pm 12 \mathrm{GeV} / \mathrm{c}^{2}$, as obtained from a simple average of the CDF [16] and the D0 [17] measurements, the Standard Model predicts $R_{b}^{0}=0.2155 \mp$ 0.0005 [15]. This number is about 2.3 standard deviations lower than our measurement, assuming $R_{c}=0.172$.

In addition, the variation of $R_{b}$ between the on peak and off peak energies has been measured. The values

$$
\begin{aligned}
& \frac{R_{b}(89.49 \mathrm{GeV})}{R_{b}(91.25 \mathrm{GeV})}=0.982 \pm 0.015 \\
& \frac{R_{b}(93.08 \mathrm{GeV})}{R_{b}(91.25 \mathrm{GeV})}=0.997 \pm 0.016
\end{aligned}
$$

have been found, in agreement with the Standard Model prediction of 0.997 and 0.998 respectively.

\section{Acknowledgements}

We are greatly indebted to our technical collaborators and to the funding agencies for their support in building and operating the DELPHI detector, and to the members of the CERN-SL Division for the excellent performance of the LEP collider. 


\section{References}

[1] ALEPH Collaboration, D. Buskulic et al., Phys. Lett. B313 (1993) 535.

[2] ALEPH Collaboration., D. Buskulic et al., Phys. Lett. B313 (1993) 549.

[3] DELPHI Collaboration, P. Abreu et al., Z. Phys. C66 (1995) 323.

[4] L3 Collaboration, O. Adriani et al., Phys. Lett. B307 (1993) 237.

[5] OPAL Collaboration, P.D. Acton et al., Z. Phys. C65 (1995) 17.

[6] The LEP collaborations, Combination of Heavy Flavour Electroweak Measurements at LEP, Publication in preparation.

[7] DELPHI Collaboration, P. Abreu et al., Z. Phys. C65 (1995) 555.

[8] DELPHI Collaboration, P. Aarnio et al., Nucl. Inst. Meth. A303 (1991) 233.

[9] DELPHI Collaboration, P. Abreu et al., Performance of the DELPHI detector, CERN-PPE/95-194, submitted to Nucl. Inst. Meth.

[10] N. Bingefors et al., Nucl. Inst. Meth. A328 (1993) 447.

[11] T. Sjöstrand et al., in "Z physics at LEP 1", CERN 89-08, CERN, Geneva, 1989; Comp. Phys. Comm. 39 (1986) 347.

[12] G. Borisov and C. Mariotti, Fine Tuning of Track Impact Parameter Resolution of the DELPHI Detector, to be published in Nucl. Inst. Meth.

[13] F. Caravaglios and G. Ross, Phys. Lett. B346 (1995) 159.

[14] P. Billoir et al., Nucl. Inst. Meth. A360 (1995) 532.

[15] D. Bardine et al., ZFITTER: An Analytical Program for Fermion Pair Production in $e^{+} e^{-}$Annihilation, CERN-TH 6443/92 (May 1992).

[16] CDF Collaboration, F. Abe et al., Phys. Rev. Lett. 74 (1995) 2626.

[17] DØ Collaboration, S. Abachi et al., Phys. Rev. Lett. 74 (1995) 2632. 